\title{
A Theory of the Evolution of Social Power: Natural Trajectories of Interpersonal Influence Systems along Issue Sequences
}

\author{
Noah E. Friedkin, Peng Jia, Francesco Bullo \\ University of California, Santa Barbara
}

Abstract: This article reports new advancements in the theory of influence system evolution in small deliberative groups, and a novel set of empirical findings on such evolution. The theory elaborates the specification of the single-issue opinion dynamics of such groups, which has been the focus of theory development in the field of opinion dynamics, to include group dynamics that occur along a sequence of issues. The theory predicts an evolution of influence centralities along issue sequences based on elementary reflected appraisal mechanisms that modify influence network structure and flows of influence in the group. The new empirical findings, which are also reported in this article, present a remarkable suite of issue-sequence effects on influence network structure consistent with theoretical predictions.

Keywords: opinion dynamics; network evolution; influence centrality

Citation: Friedkin, Noah E., Peng Jia, and Francesco Bullo. 2016. "A Theory of the Evolution of Social Power: Natural Trajectories of Interpersonal Influence Systems along Issue Sequences." Sociological Science 3: 444-472.

Received: November 7, 2015

Accepted: February 4, 2016

Published: June 21, 2016

Editor(s): Jesper Sørensen, Delia Baldassarri

DOI: $10.15195 /$ v3.a20

Copyright: (C) 2016 The Author(s). This open-access article has been published under a Creative Commons Attribution License, which allows unrestricted use, distribution and reproduction, in any form, as long as the original author and source have been credited. (C) (i)
$\mathrm{T}$ HE analysis of static social networks is an advanced field of work. Among its important contributions are formalizations of individuals' structural centralities in social networks and empirical investigations of the association of structural centrality with interpersonal influence (Katz, 1953; Hubbell, 1965; Freeman, 1979; Bonacich, 1987; Friedkin, 1991). Over the past several decades, there has been a paradigm shift from comparative-static analysis to a dynamic analysis of interpersonal influence mechanisms that unfold on a social network. A vibrant interdisciplinary field of opinion dynamics has emerged on the implications of various postulated mechanisms of interpersonal influence. ${ }^{1}$ In this new paradigm, individuals' power and influence are epiphenomena of the process of interpersonal influence that unfolds in the influence network assembled on a specific issue. An early expression of this approach is Friedkin's (1991) analysis in which a classic suite of individual centrality measures appear as emergent implications of an influence process, as opposed to static structural properties of individuals' structural positions in an arbitrary network. Thus, influence centrality has been defined as the total (direct and indirect) relative influence of each individual's initial opinion to the settled opinions of a group's members on a specific issue. The work that we report in this article is a further contribution to the group dynamics foundation of social influence and power. It extends the approach to dynamics that alter influence centralities. The assumption of a static network is relaxed and attention is shifted from single-issue dynamics (the current predominant focus of work) to dynamics that alter the influence network of a group along a sequence of issues. Issue sequences allow an evolution of group members' influence centralities, and the questions that we address are whether such evolution occurs and can be predicted. 
A prevalent feature of social organizations are the small deliberative groups that are assembled in them, whose members have been authorized to deal with issues in particular domains. In universities, such groups appear in the regular meetings of committees and department faculty. In our public school districts, elected or appointed School Boards are responsible for the provision and maintenance of educational activity. In our court system, deliberative groups include grand juries, federal appellate panels of judges, and the Supreme Court. In our Executive branch and Congress, such groups include various standing policy bodies and committees that generate advice and decisions on particular types of issues. In the large organizations of our market economy, such groups include Boards of Directors, and other bodies with decision making and advisory roles on issues that arise within restricted domains of these organizations' resource allocations, internal policies, and market related activities. In general, in most large organizations, small groups are assembled with a membership roster that is stable over some specified period time to which issues are referred that fall within a pre-specified domain. Because such groups deal with a sequence of issues, the properties of a group (its culture and power structure) may evolve over its issue sequence according to natural social processes.

We analyze a "reflected appraisal" process (Cooley, 1902; Friedkin, 2011; Jia et al., 2015) in which an individual's influence centrality on an issue alters his or her expectation of future group-specific influence on issues. With this mechanism, individuals' levels of closure-openness to interpersonal influence are group-specific social constructions, as opposed to fixed personality characteristics, that depend on their influence centralities on prior issues. Success in influencing group issue outcomes elevates the individual's self-weight (encourages opinion assertiveness, confidence, and intransigence). Failure in influencing group issue outcomes dampens the individual's self-weight (encourages opinion silence, uncertainty, or accommodation). We believe that this elementary mechanism is ubiquitous in deliberative groups that regularly meet to render recommendations or decisions, and we show that it may have startling implications for the evolution of a group's interpersonal influence system along a sequence of issues. We report signals of these implications in an experiment on 30 small groups assembled to discuss 15 choice-dilemma issues on which opinions are formed about the minimum level of confidence (i.e., a value in the $[0,1]$ interval) required to accept a risky option with a high payoff over a less risky option with a low payoff. The scope conditions of the mechanism and its implications will be discussed.

\section{Contribution and Organization}

This article contributes new theory on the evolution of influence network structure and new empirical findings on the temporal trajectories of structural features of influence networks along an issue sequence. With longitudinal data collected in experiments on 30 groups dealing with sequences of 15 issues, we report a suite of empirical findings on aspects of group evolution. The key finding is that, along an issue sequence composed of $s=1,2, \ldots, 15$ issues, a cumulative history of prior relative influence centralities is built up to issue $s$ that affects the behaviors of 
individuals and the influence systems in which they are embedded on issue $s+1$ of the sequence. This effect increases along the issue sequence.

The article is organized as follows. We start with several illustrations of the implications of the model. With these illustrations in mind, a reader may choose to skip the section on mathematical foundations, move directly to the sections that report the experimental design and empirical findings, and then return to the mathematics. In the article's online supplement, we provide a computational analysis of the reflection mechanism's implications (Section S1), a description of the attractor system toward which the group evolves (Section S2), information on the experiment's protocol (Section S3), and a discussion of the model's application to the literature on groupthink (Section S4).

\section{Illustrations}

Several simulations of the model serve to describe the startling predictions that motivated the collection of data on group behavior along issue sequences. Answers to questions on the mathematical foundations of these illustrations (e.g., the definition of an influence system, the definition of a stable strongly connected structure of interpersonal influences, the definition of random influence structures, and the specifications of the postulated dynamics) may be found in the mathematical foundations section.

In the issue-specific influence systems that a group constructs on each issue of an issue sequence, $s=1,2, \ldots$, the reflection mechanism posits that individuals' levels of closure to influence on issue $s+1$ of the sequence adjust in correspondence with their prior relative influence centralities on issue $s$ of the sequence. In a stable, strongly-connected structure of interpersonal influences, individuals' adjustments of their levels of closure to influence operate to dampen or elevate the strength of these interpersonal influences. A realization of the predicted evolution in this case is illustrated in Figure 1. One member moves toward complete closure to influence and maximal influence centrality, and all other members move toward complete openness to influence and minimal influence centrality. Figure 2 is based on the same $s=1$ initial conditions of Figure 1. As in Figure 1, the configuration of $i \rightarrow j, i \neq j$ allocations of influence is fixed along the issue sequence, but here random realizations of the values of these allocations are introduced on each issue $s=2,3, \ldots$ Figure 3 is based on the same $s=1$ initial conditions of Figure 1 . But here, nothing is fixed except the strong connectivity property of the influence network: the strongly connected configuration of $i \rightarrow j, i \neq j$ allocations of influence is now a random structure, and the values of influence allocations are random values on each issue $s=2,3, \ldots$. Who an individual $i$ allocates influence to is now an issue-specific variable. When the reflection mechanism unfolds in such an unstable structure, the influence system repetitively approaches the generic attractor system, but the approach is not necessarily to states with the same dominant individual. Severe disturbances may reorganize the system as, for example, when the disturbance presents a concentration of allocated influences on an individual who is near completely open to influence and who allocates little or no influence to the near completely closed member of the group. Such reorganizations will continue 

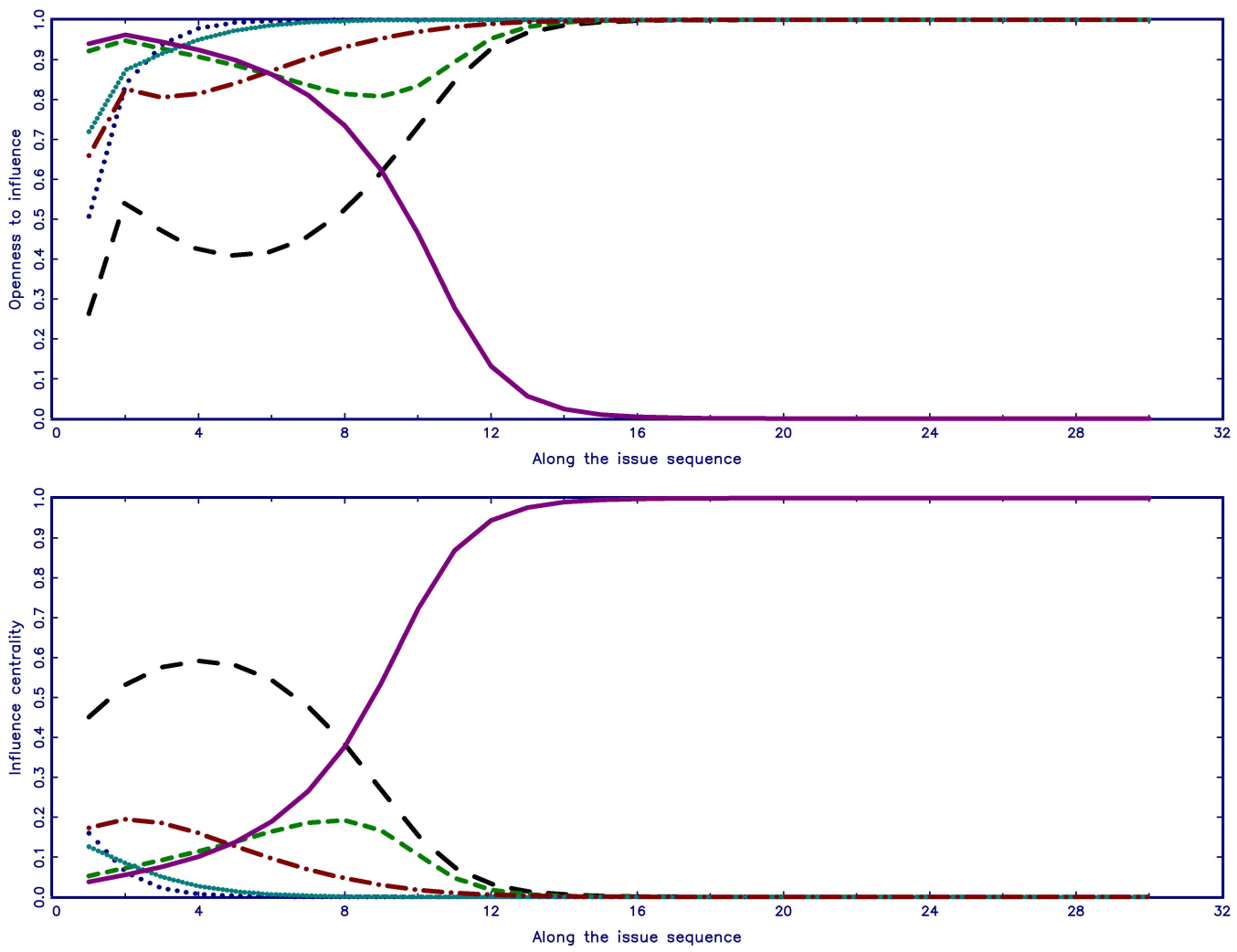

Figure 1: Illustration of the predicted evolution in a $n=6$ group with a stable strongly connected structure of relative interpersonal influences. Along the sequence of issues $s=1,2, \ldots$, the reflection mechanism alters members' levels of openness to influence on issue $s+1$ in correspondence with their prior influence centrality on issue $s$ of the sequence. The top panel plots the changing levels of individuals' levels of openness to influence. One member moves toward complete closure and all others move toward complete openness. These adjustments elevate or dampen the strength of individuals' allocated influences to others. The bottom panel plots the associated changes of influence centrality. One member moves toward maximal centrality and all others move toward minimal centrality.

so long as the configuration of "who has direct influence on whom" is subject to severe disturbances. In the aftermath of each structural disturbance, the reflection mechanism operates to return the influence system to a trajectory toward the generic outcome illustrated in Figures 1 and 2, that is, toward an influence system in which one individual (but not necessarily the same individual) has maximal centrality and all others minimal centrality.

In the theory that underlies these illustrations, heterogeneous levels of resistance to opinion change prohibit reaching an exact consensus on issues. The theory makes no assumption about the subjective importance of small versus large differences of opinion, and no homogeneity assumptions on individuals' allocations of influence. Its assumptions focus on influence system mechanisms. This focus does not diminish the importance of social structure. We cannot understand the implications of influence system mechanisms without attending to the network structure in which the mechanisms are unfolding, and we cannot understand the implications of a 

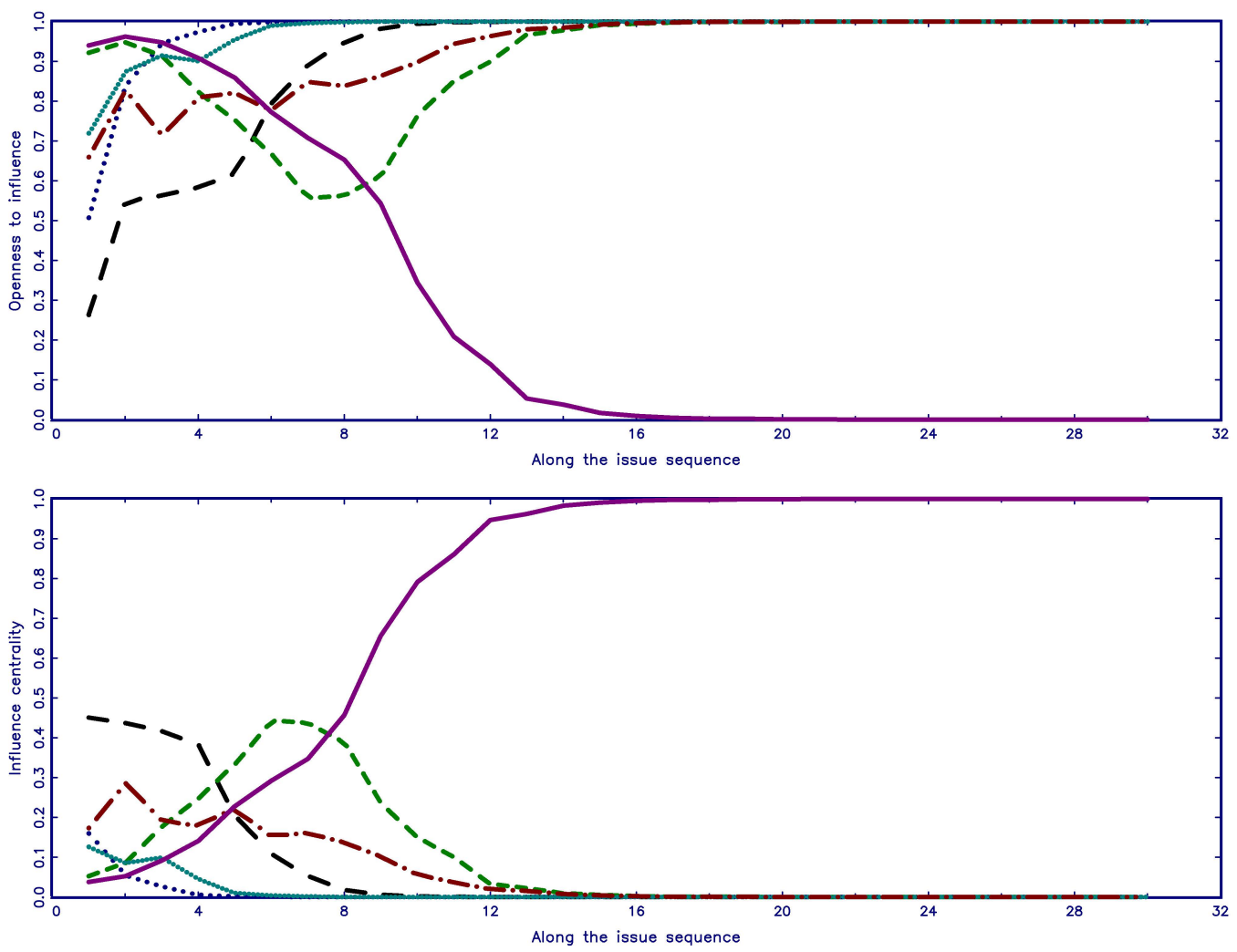

Figure 2: Illustration of the predicted evolution in a $n=6$ group with the same initial conditions of the Figure 1 group. The group's strongly connected configuration of relative interpersonal interpersonal influences is stable as in Figure 1, but here the values of allocated influences are randomly disturbed along the issue sequence on each issue $s=2,3, \ldots$

network structure without attending to the mechanisms that are unfolding on it. The realizations of the mechanisms investigated in this article are diverse because the network structures in which the mechanisms unfold are diverse. Special cases of influence systems exist in which the reflection mechanism does not generate an evolution to a structure with one maximally central member. For example, one such special case is the perfect peer group with a complete influence network in which all allocated influences to others are of equal strength on every issue of the group's issue sequence. We locate our interest on the fundamental assumption that applies to all possible realizations of the reflection mechanism. Do individuals, in fact, tend to adjust their levels of closure to influence on issue $s+1$ of an issue sequence $s=1,2, \ldots$ in response to their prior influence centrality on issue $s$ of the sequence or, more generally, to their evolving average influence centralities on all previous issues? We will also investigate whether this mechanism is associated with an orderly evolution of observed properties of the group's influence network along an issue sequence. 

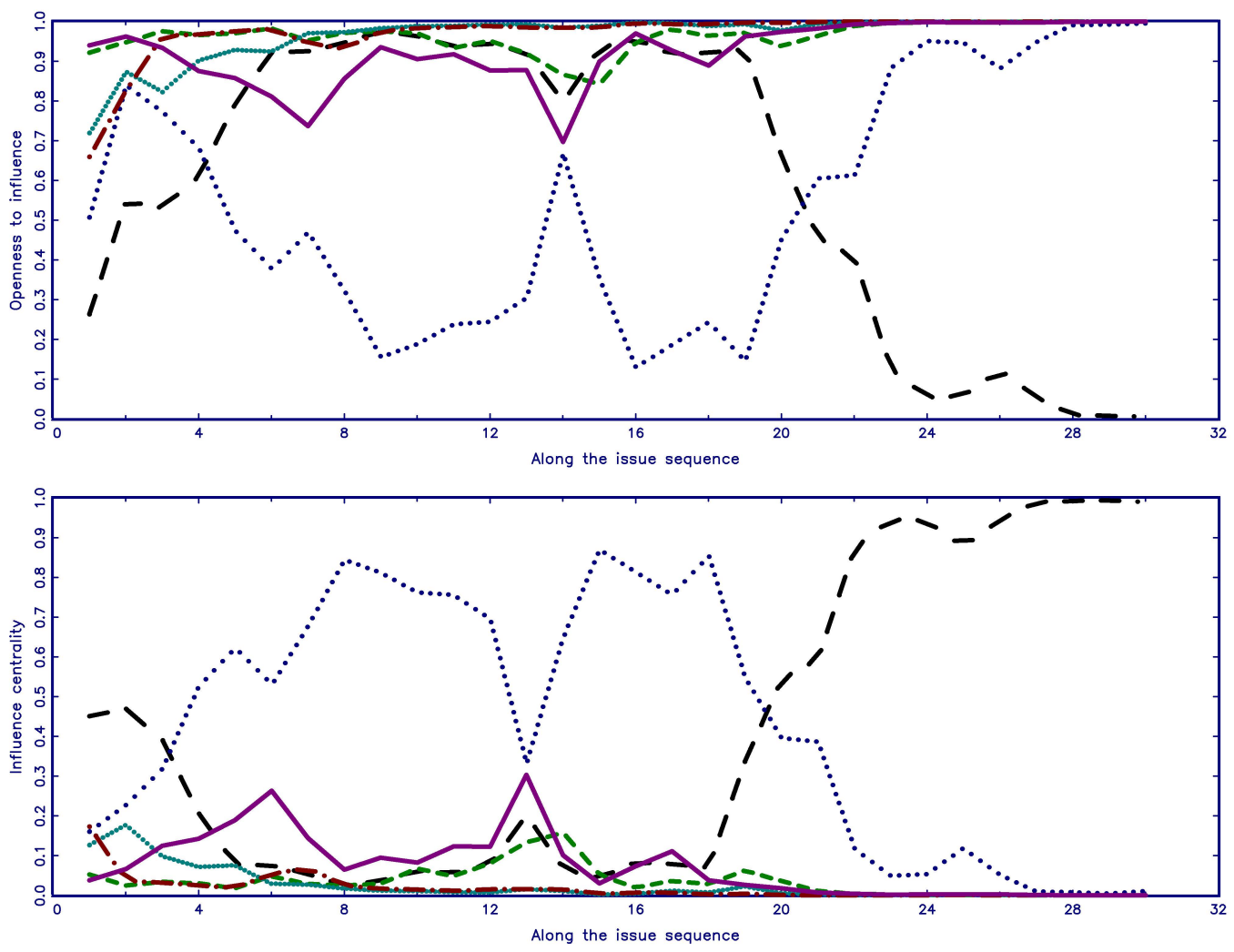

Figure 3: Illustration of the predicted evolution in a $n=6$ group with the same initial conditions of the Figure 1 group. The group's strongly connected configuration of relative interpersonal interpersonal influences and the values of allocated influences on it are both randomly disturbed along the issue sequence on each issue $s=2,3, \ldots$

\section{Formalization}

The formalization defined in this section is constructed on the Friedkin-Johnsen (2011) model of single-issue opinion dynamics in a group of two or more individuals. In this model, individuals' influence centralities are derived implications of the influence process that unfolds on the influence network of the group. The derived influence centralities correspond to the relative contributions of each group member's initial opinion on a specific issue to the model's predicted equilibrium opinions on the issue. We begin with the mathematics of this model and then introduce the reflection mechanism that alters the influence network of the group along an issue sequence.

\section{Influence process on a specific issue}

The Friedkin-Johnsen (F-J) model of interpersonal influence systems is a generalization of the seminal French-Harary-DeGroot work on opinion dynamics (French, 1956; Harary, 1959; DeGroot, 1974). See Friedkin and Johnsen (2011) for its motivations and analysis including relevant theorems and empirical supports. Here 
we present only the essential features of the model that allow the derivation of individuals' influence centralities on a specific issue.

In its generalization, the F-J model allows "memory" in the form of an ongoing anchorage on initial positions and, by implication, an anchorage on the antecedent conditions of individuals' initial issue positions, e.g., their fixed interests and circumstances. Thus, the French-Harary-DeGroot process was opened to allow for end states of unresolved interpersonal disagreement on an issue in the context of influence network structures that previously had one inevitable outcome-consensus. The generalization retains the French-Harary-DeGroot formalization of social influence as a positive finite resource that is possessed by each individual $i$ of a group of $n$ individuals and distributed by $i$ to self and to particular other members of the group, $0 \leq w_{i j} \leq 1, \sum_{j=1}^{n} w_{i j}=1$, for each $i=1, \ldots, n$. Each self-weight $w_{i i}$ determines the weight that is allocated to others as an aggregate, $w_{i i}=1-\sum_{j \neq i}^{n} w_{i j}$. The aggregate relative influence that individual $i$ allocates to other individuals, $1-w_{i i}$, describes the extent to which an individual is inner- or other-directed on a specific issue.

The F-J interpersonal influence system is specified by a discrete time process of iterated weighted averaging of individuals' opinions on an issue,

$$
x_{i}(k+1)=a_{i i} \sum_{j=1}^{n} w_{i j} x_{j}(k)+\left(1-a_{i i}\right) x_{i}(0), \quad(i=1, \ldots, n ; k=0,1, \ldots),
$$

where for all $i, a_{i i}=1-w_{i i}$, and $x_{i}(0) \in \mathcal{R}$ is the initial opinion of the group member on an issue. For an individual $i$ with $w_{i i}=1$, the individual is completely closed to interpersonal influence on the issue, and the fixed position of the individual is the individual's initial opinion. For an individual $i$ with $w_{i i}=0$, the individual is completely open to interpersonal influence on the issue, and at each time $k$ the individual is strictly oriented to the opinions of those group members to whom $i$ has allocated influence. For an individual $i$ with $0<w_{i i}<1$, the initial opinion of the individual makes a continuing direct contribution $1-a_{i i}=w_{i i}$ to the time $k+1$ influenced position of $i$, depending on the extent to which the individual is open or closed to interpersonal influence. In this case, the $a_{i i} w_{i i}>0$ value is the weight that $i$ allocates to his or her own updated opinions during the process of opinion change. This weight corresponds to $i$ 's resistance to opinion change per se, and its maximal value is 0.25 . The system of equations for the influence system on a specific issue is described by the matrix equation

$$
\mathbf{x}(k+1)=\mathbf{A W x}(k)+(\mathbf{I}-\mathbf{A}) \mathbf{x}(0), \quad(k=0,1, \ldots),
$$

where $\mathbf{A}=\left[a_{i i}\right]$ is a diagonal matrix, with $a_{i i}=1-w_{i i}$ values on the main diagonal and zeros elsewhere, and $\mathbf{I}$ is the identity matrix (with ones on the main diagonal and zeros elsewhere).

\section{Total influences and influence centralities}

Equation 1 defines the direct influences, at each time $k$, on each group member's opinion. Indirect interpersonal influences on an individual arise from the repetitive 
responses (information integration activity) of individuals to the changing opinions of those to whom they have allocated direct influence. Thus, an indirect influence arises when the opinion of a particular group member $j$, to whom $i$ has allocated influence, has been affected by some other group member $u$. At each time $k$, a matrix of direct and indirect influences exists, $\mathbf{V}(k)=\left[v_{i j}(k)\right]$, that defines the relative net influence of each group member $j$ 's initial opinion on the opinion of $i$ at time $k+1$,

$$
\begin{aligned}
x_{i}(k+1) & =a_{i i} \sum_{j=1}^{n} w_{i j} x_{j}(k)+\left(1-a_{i i}\right) x_{i}(0) \\
& =\sum_{j=1}^{n} v_{i j}(k) x_{j}(0),
\end{aligned}
$$

where $0 \leq v_{i j}(k) \leq 1$ for all $i$ and $j$, and $\sum_{j=1}^{n} v_{i j}(k)=1$ for all $i$. The derivation of $\mathbf{V}(k)$ may be obtained either with the matrix recursion, $\mathbf{V}(1)=\mathbf{A W}+(\mathbf{I}-\mathbf{A})$,

$$
\mathbf{V}(k+1)=\mathbf{A W V}(k)+(\mathbf{I}-\mathbf{A}), \forall k>0,
$$

or equivalently with the evolving matrix polynomial of walks in the network structure of AW,

$$
\mathbf{V}(k)=(\mathbf{A W})^{k}+\left[\sum_{i=0}^{k-1}(\mathbf{A W})^{i}\right](\mathbf{I}-\mathbf{A}), \forall k>0 .
$$

The sequence $\{\mathbf{V}(k) ; k=0,1, \ldots\}$ converges and an equilibrium exists, if and only if the $\lim _{k \rightarrow \infty}(\mathbf{A W})^{k}$ exists. There are several sufficient conditions of convergence that are associated with the particular topology of AW. The broadest sufficient condition is $\mathbf{0}<\mathbf{A}<\mathbf{I}$, which gives $\lim _{k \rightarrow \infty}(\mathbf{A W})^{k}=\mathbf{0}$, and a sequence $\{\mathbf{V}(k) ; k=$ $0,1, \ldots\}$ that converges to

$$
\mathbf{V}=\left[\sum_{k=0}^{\infty}(\mathbf{A W})^{k}\right](\mathbf{I}-\mathbf{A})=(\mathbf{I}-\mathbf{A W})^{-1}(\mathbf{I}-\mathbf{A}) .
$$

In general, when $\lim _{k \rightarrow \infty}(\mathbf{A W})^{k}$ exists, the equilibrium matrix equation for the system is

$$
\mathbf{x}(\infty)=\mathbf{A W x}(\infty)+(\mathbf{I}-\mathbf{A}) \mathbf{x}(0)=\mathbf{V} \mathbf{x}(0) .
$$

where $\mathbf{V}=\left[v_{i j}\right]$ is the derived matrix of total (direct and indirect) influences of group member $j^{\prime}$ s initial opinion on group member $i$ 's settled opinion on an issue. With $0 \leq v_{i j} \leq 1$ for all $i$ and $j$, and $\sum_{j=1}^{n} v_{i j}=1$ for all $i, v_{i j}$ is the equilibrium relative total contribution of group member $j$ 's initial opinion to the settled opinion of group member $i$. Given $\mathbf{V}$, each group member $i$ has a mean relative influence centrality, that is,

$$
C_{i}(s)=\frac{1}{n} \sum_{j=1}^{n} v_{j i}(s),
$$

where each $v_{j i}$ is the total relative influence of $i$ 's initial opinion on $j^{\prime}$ 's equilibrium opinion. These mean influence centralities sum to 1 . Eigenvector influence centrality 
appears in the special case of $\mathbf{A}=\mathbf{I}$. Page-Rank centrality appears in the special of $\mathbf{A}=\alpha \mathbf{I}, 0<\alpha<1$.

\section{Definitions of influence network topology}

The final essential component of the work to be presented is a set of definitions of influence network typology. The matrix AW is associated with a directed valued network. The set of valued directed edges of the network are the positive elements

of AW, $i \stackrel{a_{i i} w_{i j}>0}{\longrightarrow} j \forall i j$. The topology of the influence network is the configuration of these positive edges ignoring their values. Let $\mathcal{G}$ be the directed graph of AW defined on edges $i \stackrel{a_{i i} w_{i j}>0}{\longrightarrow} j$ that correspond to the subset of positive elements of AW. The powers of $\mathcal{G}^{k} k=1,2, \ldots$ correspond to walks of length $k$ in $\mathcal{G}$. The powers of $(\mathbf{A W})^{k}$ correspond to the values of all walks in the influence network. Thus, the discrete-time specification of the influence process allows the application of the highly developed body of general theorems on graphs and matrices. A determination of AW's connectivity properties is essential in the investigation of the influence system, and classificatory language is necessary to define the type of system that is being analyzed.

The most important classifications are the following. The matrix AW is in the class of nonnegative matrices for which a rich body of theorems exist. It is also, depending on $\mathbf{A}$, either stochastic $\left(a_{i i}=1\right.$ for all $\left.i\right)$, strictly substochastic $\left(a_{i i}<1\right.$ for all $i$ ), or not-strictly substochastic $\left(a_{i i}<1\right.$ for at least one but not all $\left.i\right)$. The spectral properties of such matrices are well-understood. The network associated with AW is strongly connected if for all $(i, j)$ ordered pairs of individuals there is at least one path from $i$ to $j$ and at least one path from $j$ to $i$. The matrix $\mathbf{A W}$ is reducible if it is not strongly connected and irreducible if and only if it is strongly connected. If $\mathcal{G}$ is not strongly connected, then it is reducible and must have either a unilateral, weak or disconnected topology. It is unilateral if it is not strongly connected and for all $(i, j)$ ordered pairs of individuals there is at least one path from $i$ to $j$ or at least one path from $j$ to $i$. It is weak if it is not unilateral and for all $(i, j)$ unordered pairs of individuals there exists at least one chain of edges that connects them ignoring the direction of the edges, e.g., $i \rightarrow k \leftarrow u \leftarrow v \rightarrow j$. It is disconnected if it is not weak. ${ }^{2}$

\section{Influence system evolution along an issue sequence}

From Equation 8, on each issue $s$ of an issue sequence, $s=1,2, \ldots$, each group member $i$ has a mean relative influence centrality, $C_{i}(s)$. The reflection mechanism assumption is that group members' attachments to their initial opinions on issue $s+1$ of the issue sequence elevate or dampen along an issue sequence in correspondence to their relative influence centralities on issue $s$ of the sequence. That is,

$$
1-a_{i i}(s+1)=w_{i i}(s+1)=C_{i}(s),
$$

for all $i$ and $s=1,2, \ldots$. With this mechanism, individuals' levels of closureopenness to interpersonal influence become social constructions, as opposed to fixed personality characteristics, that depend on their realized influence centralities on prior issues. Successes in influencing issue outcomes elevate self-weight (foster 
assertiveness, confidence, and intransigence). Failures in influencing issue outcomes dampen self-weight (foster silence, uncertainty, and accommodation). The updated $1-a_{i i}(s+1)$ are associated with the $w_{i j}(s+1), i \neq j$, as follows,

$$
w_{i j}(s+1)=a_{i i}(s+1) r_{i j}(s+1)+1-a_{i i}(s+1),
$$

where $r_{i j}(s+1)$ is the issue-specific relative interpersonal weight allocated by $i$ to $j$, $r_{i i}(s+1)=0,0 \leq r_{i j}(s+1) \leq 1$, and $\sum_{j=1}^{n} r_{i j}=1$ for all $i$ and all $i \neq j$. The matrix equation of this association is $\mathbf{W}(s+1)=\mathbf{A}(s+1) \mathbf{R}(s+1)+\mathbf{I}-\mathbf{A}(s+1)$, for all $s$.

When the individual-level adjustments of the reflection mechanism are occurring among individuals embedded in a strongly connected influence system, they may dramatically alter the behavior of the entire system. On each issue, the influence network that is assembled by the group may or may not operate to importantly modify group members' opinions on the issue. Whether it does or not, depends on its members' issue-specific levels of closure to influence, the configuration of interpersonal allocations of influence in the group, and the relative weights of these allocations. These components of the influence system determine the direct and indirect flows of interpersonal influence and trajectories of opinion change. The reflection mechanism reorients the classic focus of social network analysis on the structural topology of the connectivity of individuals to a focus on the implications of node loops. An influence network is composed of nodes that are individuals, and directed $i \rightarrow j$ lines that indicate an allocation of influence from $i \rightarrow j$. The lines have values that specify how much influence is allocated by $i$ to $j$. The unusual feature of this social network is that $i \rightarrow i$ directed lines and their values are meaningful and important. The values of the network's loops specify individuals' levels of attachment to their initial positions on a specific issue, and their resistances to opinion change. Changes of value on a particular $i \rightarrow i$ loop strengthen or dampen all influence flows that involve $i$, and in a strongly connected system (i.e., a system in which all members directly or indirectly influence each other) all influence flows among all members are affected by such changes. Thus, if the loop values evolve along the issue sequence of a group, then (except in rare special cases) the relative influence centralities of individuals will also adjust.

The computational analyses reported in the online supplement (Section S1) indicate that, with rare exceptions, in strongly connected influence networks the trajectories of group members' self-weights differentiate: $n-1$ self-weights are dampened to 0 and one member's self-weight is elevated to 1 . The system-level consequence is an influence system with a trajectory, as a system, toward a state in which the influence centrality of one individual is elevated to value near 1, and influence centralities of the other $n-1$ members are dampened to values near 0 . Thus, the trajectory is toward a system (attractor) that generates a consensus on the initial opinion, whatever it may be, of the one member whose self-weight has been elevated to a state of complete closure to interpersonal influence. The properties of this attractor system are described in the online supplement (Section S2).

The mechanism defined in Equation 9 assumes that individuals have a memory of their $s$ influence centrality but no memory of their influence centralities on issues 
prior to $s$, i.e., $s-1, s-2, \ldots, 1$. This assumption may be relaxed as follows,

$$
1-a_{i i}(s+1)=w_{i i}(s+1)=\frac{1}{s} \sum_{t=1}^{s} C_{i}(t),
$$

where $T_{i}(s)=\frac{1}{s} \sum_{t=1}^{s} C_{i}(t)$ is the individual's time-average influence centrality. For the $n$ individuals of the group, these time-averaged influence centralities sum to 1 at each $s$. We will present findings on the usefulness of this measure.

\section{Experiment Design and Measurement Models}

The research question is whether or not individuals' level of closure-openness to interpersonal influence adjusts in response to their prior influence centralities in a group that is considering a sequence of issues. Such adjustments assume that each group member has a veridical appraisal of their own relative influence centrality on each issue of the sequence. It should also be obvious that detection of the mechanism depends on an accurate specification of single-issue opinion dynamics and validated measurement models of the theoretical constructs of the specification.

Since its inception, the development of the F-J specification has been coupled with empirical research on its predictions. This work has drawn on a standard pre- and post-discussion design with which social psychologists have investigated choice shifts (changes in group members' mean initial and final opinion) in small groups on choice-dilemmas; see Friedkin and Jonsen (2011) for a review of this literature. Choice dilemmas require the formation of opinions on the minimum level of confidence (i.e., a value in the $[0,1]$ interval) required to accept a risky option with a high payoff over a less risky option with a low payoff. In experiments conducted on choice dilemmas subjects are randomly assigned to small groups (e.g., dyads, triads, or tetrads), an issue is posed, subjects privately record their initial positions on the issue, a group discussion on the issue is opened, and the discussion is ended when the group has reached a state of settled opinions. Friedkin and Johnsen elaborated the design with a post-discussion self-report by each subject of the extent to which his or her opinion was influenced by other group members and the relative influence of those members (if any) whose opinions influenced them.

The details of the F-J enhanced design have been reviewed and reported in previous publications (Friedkin and Johnsen, 2011). Here we employ the same design to reduce the probability of a null finding on the reflection mechanism based on an important misspecification of single-issue opinion dynamics or faulty measures of the theoretical constructs of the specification. The pre-existing body of positive findings on the F-J predictions of group members' final opinions supports its single-issue opinion dynamics specification and construct measurement models. These predictions have been obtained as follows. The design provides a measure of $\mathbf{x}(0)$ (the group members' initial opinions on an issue) and a measure of $\mathbf{W}$ (the group's matrix of influence allocations on the issue). The group's matrix of total influences $\mathbf{V}$ is derived strictly on the basis of $\mathbf{W}$. With no fitting, the predicted final opinions of the group's members are $\mathbf{x}(\infty)=\mathbf{V} \mathbf{x}(0)$. Thus, we have a pre-existing basis of confidence in the $\mathbf{V}$ matrix upon which our measure of group members' 
Table 1: Prediction of an individual's final opinion on an issue. Opinions are scaled $0-100$. Standard errors are in parentheses.

\begin{tabular}{|c|c|c|c|}
\hline & (a) & (b) & (c) \\
\hline F-J prediction & & $\begin{array}{c}0.897^{*} \\
(0.018)\end{array}$ & $\begin{array}{c}1.157^{*} \\
(0.032)\end{array}$ \\
\hline Initial opinion & & & $\begin{array}{r}-0.282^{*} \\
(0.031)\end{array}$ \\
\hline Constant & $\begin{array}{c}58.975^{*} \\
(1.550)\end{array}$ & $\begin{array}{c}5.534 \\
(1.176)\end{array}$ & $\begin{array}{r}6.752^{*} \\
(1.124)\end{array}$ \\
\hline Log likelihood & -8579.835 & -7329.003 & -7241.097 \\
\hline
\end{tabular}

Notes: $* p \leq 0.001$; balanced random-intercept multilevel longitudinal design; maximum likelihood estimation with robust standard errors; $n=1,800$.

influence centralities is constructed $C_{i}(s)=\frac{1}{n} \sum_{j=1}^{n} v_{j i}(s)$ on each issue of an issue sequence $s=1,2, \ldots$

The subjects involved in present experiment were recruited from the undergraduate population of a large USA West Coast university. Our findings are based on 30 groups, each with 4 members, dealing with a sequence of 15 (randomly ordered) choice dilemma issues under either high or low pressure to reach consensus. Random assignment of subjects to groups eliminates bias in the composition of the groups. Random issue orders eliminate bias of issue order. The online supplement (Section S3) describes these issues, the measure model of $\mathbf{W}$, and the consensus pressure conditions. The data structure is a multilevel balanced longitudinal design. ${ }^{3}$

\section{Specification check}

This new dataset triples the length of the longest sequence that has been previously examined. In an abundance of caution, we assess whether this dramatic increase in the length of the issue sequence alters the performance of the F-J model. If the model's derived matrices of total influences do not present satisfactory predictions of individuals' final opinions and opinion changes on single issues, then there is no credible basis for investigating the evolution of the influence system, in terms of this model, along a lengthy issue sequence.

Table 1 confirms that the F-J predictions perform in this new experiment as they have in previous experiments. The Pearson product-moment correlation $\rho$ of observed and predicted final opinions is $\rho=0.872$. The correlation of observed opinion changes, $x_{i}(\infty)-x_{i}(0)$, and predicted opinion changes, $\hat{x}_{i}(\infty)-x_{i}(0)$, is $\rho=0.811$. An alternative predictor of opinion changes, also directly based on the model, is $a_{i i}\left(\sum_{j=1}^{n} w_{i j} x_{j}(\infty)-x_{i}(0)\right)$. This predictor is also correlated $(\rho=0.915)$ with observed opinion changes. 


\section{Measures}

For each group, on each issue, the foundations of all measures are the $4 \times 1$ vector of pre-discussion initial opinions $\mathbf{x}(0)$, the $4 \times 1$ vector of post-discussion final opinions $\mathbf{x}(\infty)$, and the $4 \times 4$ matrix of relative influence allocations $\mathbf{W}$ that were obtained on each issue of the sequence (online supplement, Section S3).

Initial and final opinions. The measure of opinions is a $0-100$ percent scale of the minimum level of confidence required to accept a risky option with a high payoff over a less risky option with a low payoff. For each subject $i$, the data includes measures of a subject's initial and final opinions on each issue $s=1, \ldots, 15$ of the issue sequence $\left(x_{i s}(0), x_{i s}(\infty)\right)$. On each issue, the group's range of initial opinions is given by the maximum and minimum values of the group members' initial opinions about the issue. The size of the initial-opinion-range varies between 0 and 100, with a median size 50, mean size 48 , and standard deviation 24 .

Issue-specific group influence allocation matrix and network. The measure of a group's influence allocation matrix $\mathbf{W}$ on issue $s$ of the group's issue sequence is determined by each subject's post-discussion allocation of 100 units of influence to indicate the relative weights of the direct interpersonal influences on their issuespecific opinions during the discussion. Dividing the number of units that subject $i$ allocates to subject $j$ by 100 gives a distribution of relative weights $w_{i 1}, w_{i 2}, w_{13}, w_{i 4}$ for each $i$, and in turn the issue-specific $\mathbf{W}$ influence matrix of the group. The main-diagonal values of this matrix are the measure of each subject's level of closure to influence on a specific issue and the subject's ongoing attachment to his or her initial opinion. The $a_{i i}=1-w_{i i}$ value is the measure of each subject's openness to influence, and the aggregate weight allocated to other group members. The measure of the AW matrix, which determines the flows of direct and indirect influence, is strictly based on $\mathbf{W}$.

Influence centralities. Given $\mathbf{W}$, the model's assumption $a_{i i}=1-w_{i i}$ for all $i$ determines the main-diagonal values of the model's A construct. Given A and W, the model's matrix of total (direct and indirect) relative influences $\mathbf{V}$ is determined (Equation 5 ). The mean of column $j$ of $\mathbf{V}$ is the measure of $j$ 's issue-specific influence centrality, $C_{j}=\frac{1}{n} \sum_{i=1}^{n} v_{i j}$ for all $j$. These centrality values are nonnegative values that sum to 1 . Along the issue sequence, $s=1,2, \ldots$, the time-average influence centrality of individual $j$ is given by $T_{j}(s)=\frac{1}{s} \sum_{t=1}^{s} C_{j}(t)$, and these centralities are also nonnegative values that sum to 1 at each $s$.

Observed and predicted time to opinion settlement. The measure of the observed time (minutes) to a settled state of opinions is the length of time from the opening of a group discussion on a specific issue to the time at which the group reported their final opinions on the issue. The observed times vary between 3 and 27 minutes, with a median time 11, mean time 12, and standard deviation 5 . The predicted time to opinion settlement is based on the number of opinion updates $k$ required to reach a near settled state of opinion on issue $s$. The convergence of the influence system to equilibrium opinions on a specific issue depends on the convergence of $(\mathrm{AW})^{k}, k=1,2, \ldots$. The convergence time predictor is $\ln (\mathrm{stab})$, where stab is the number of iterates required to reduce the sum of absolute values of the elements of $\alpha\left[(\mathbf{A W})^{k}-(\mathbf{A W})^{k+1}\right], \alpha=(1.0 e-6)$ to less than $1-(1.0 e-6)$. 
Additional structural metrics. Additional structural metrics having theoretical bearing on the postulate that influence systems evolve along an issue sequence are also defined and investigated. These additional structural metrics, defined on AW, include the value of its dominant eigenvalue, its trace (the sum of its main-diagonal values), the density of its instances of nonzero interpersonal influence, and its connectivity category (disconnected, weak, unilateral, or strong). The substantive implications of their evolution are addressed as each is introduced in the findings presented below.

\section{Findings}

This section is organized in two subsections that attend to the individual-level reflection mechanism and group-level influence structure, respectively. At the individual-level, the 1,800 individual-level observations are nested in 30 groups, and the theory's predictions of their behavior along the sequence of 15 issues are evaluated. At the group-level, the behavior of the 450 group influence structures along the issue sequence are investigated.

\section{Reflection mechanism}

Our key findings are presented in this section. The question we assess is whether or not individuals' levels of closure to influence on issue $s+1$ adjust along the issue sequence $s=1,2 \ldots$ in correspondence with their prior influence centralities. Two measures of influence centrality are evaluated: the individual's prior issue-specific centrality $C_{i}(s)$ and prior time-averaged cumulative centrality $T_{i}(s)=\frac{1}{s} \sum_{t=1}^{s} C_{i}(t)$. Table 2 shows that both measures predict individuals' issue-specific levels of closure on the next issue. The effect of $C_{i}(s)$ on $w_{i i}(s+1)$ is constant along the issue sequence. The effect of $T_{i}(s)$ on $w_{i i}(s+1)$ increases along the sequence $0.404+0.095 s$, $s=1,2, \ldots, 15$.

\section{Evolution of influence centrality}

The theory posits that an implication of the reflection mechanism is the evolution of influence centrality along the issue sequence. The predicted evolution elevates the influence centrality of one individual and dampens the influence centralities of all others. With structural disturbances, the postulated trajectory may be upset by dramatic alterations of the configuration of influence allocations (e.g., a change from a strongly connected network topology to a weak or disconnected topology), but the predicted evolution is expected to reassert itself as a process in the immediate aftermath of such disruptions.

On average, we expect a signal that is consistent with the mechanism amidst the noise of disturbances. The existence of the signal rests on the assumption of a strong association of individuals' issue $s$ levels of closure and their issue $s$ influence centralities. This association depends on the extent to which the main-diagonal values of the AW matrix of allocated influences affect the values of all flows of influence among a group's members, and in the small groups of this study, it is 
Table 2: Prediction of an individual's level of closure to influence $w_{i i}(s+1)$ based on the individual's prior centrality $C_{i}(s)$ and time-averaged cumulative centrality $T_{i}(s)=\frac{1}{s} \sum_{t=1}^{s} C_{i}(t)$. Standard errors are in parentheses.

\begin{tabular}{|c|c|c|c|}
\hline & (a) & (b) & (c) \\
\hline$C_{i}(s)$ & & $\begin{array}{c}0.336^{*} \\
(0.104)\end{array}$ & \\
\hline$T_{i}(s)$ & & & $\begin{array}{r}0.404^{+} \\
(0.159)\end{array}$ \\
\hline$s$ & & $\begin{array}{c}0.002 \\
(0.004)\end{array}$ & $\begin{array}{r}-0.018^{*} \\
(0.005)\end{array}$ \\
\hline$s \times C_{i}(s)$ & & $\begin{array}{c}0.171 \\
(0.012)\end{array}$ & \\
\hline$s \times T_{i}(s)$ & & & $\begin{array}{c}0.095^{*} \\
(0.018)\end{array}$ \\
\hline Constant & $\begin{array}{c}0.643^{*} \\
(0.016)\end{array}$ & $\begin{array}{c}0.515^{*} \\
(0.030)\end{array}$ & $\begin{array}{r}0.498^{*} \\
(0.039)\end{array}$ \\
\hline Log likelihood & -367.331 & -327.051 & -293.656 \\
\hline
\end{tabular}

Notes: $* p \leq 0.001,+p \leq 0.01$; balanced random-intercept multilevel longitudinal design; maximum likelihood estimation with robust standard errors; $n=1,680$. An entertained $s^{2}$ effect is insignificant.

Table 3: Prediction of an individual's $C_{i}(s+1)$ influence centrality based on the individual's prior $C_{i}(s)$ centrality and time-averaged cumulative centrality $T_{i}(s)=\frac{1}{s} \sum_{t=1}^{s} C_{i}(t)$ along the issue sequence. Standard errors are in parentheses.

\begin{tabular}{|c|c|c|c|}
\hline & (a) & (b) & (c) \\
\hline$C_{i}(s)$ & & $\begin{array}{c}0.166^{*} \\
(0.042)\end{array}$ & \\
\hline$T_{i}(s)$ & & & $\begin{array}{c}0.176^{\dagger} \\
(0.072)\end{array}$ \\
\hline$s$ & & $\begin{array}{c}-0.002 \\
(0.002)\end{array}$ & $\begin{array}{r}-0.012^{*} \\
(0.002)\end{array}$ \\
\hline$s \times C_{i}(s)$ & & $\begin{array}{c}0.008 \\
(0.006)\end{array}$ & \\
\hline$s \times T_{i}(s)$ & & & $\begin{array}{c}0.049^{*} \\
(0.010)\end{array}$ \\
\hline Constant & $\begin{array}{c}0.025^{*} \\
(0.000) \\
\end{array}$ & $\begin{array}{c}0.209^{*} \\
(0.011) \\
\end{array}$ & $\begin{array}{r}0.206^{*} \\
(0.018) \\
\end{array}$ \\
\hline Log likelihood & 1054.434 & 1098.319 & 1141.263 \\
\hline
\end{tabular}

Notes: $* p \leq 0.001,+p \leq 0.01$; balanced random-intercept multilevel longitudinal design; maximum likelihood estimation with robust standard errors; $n=1,680$. 


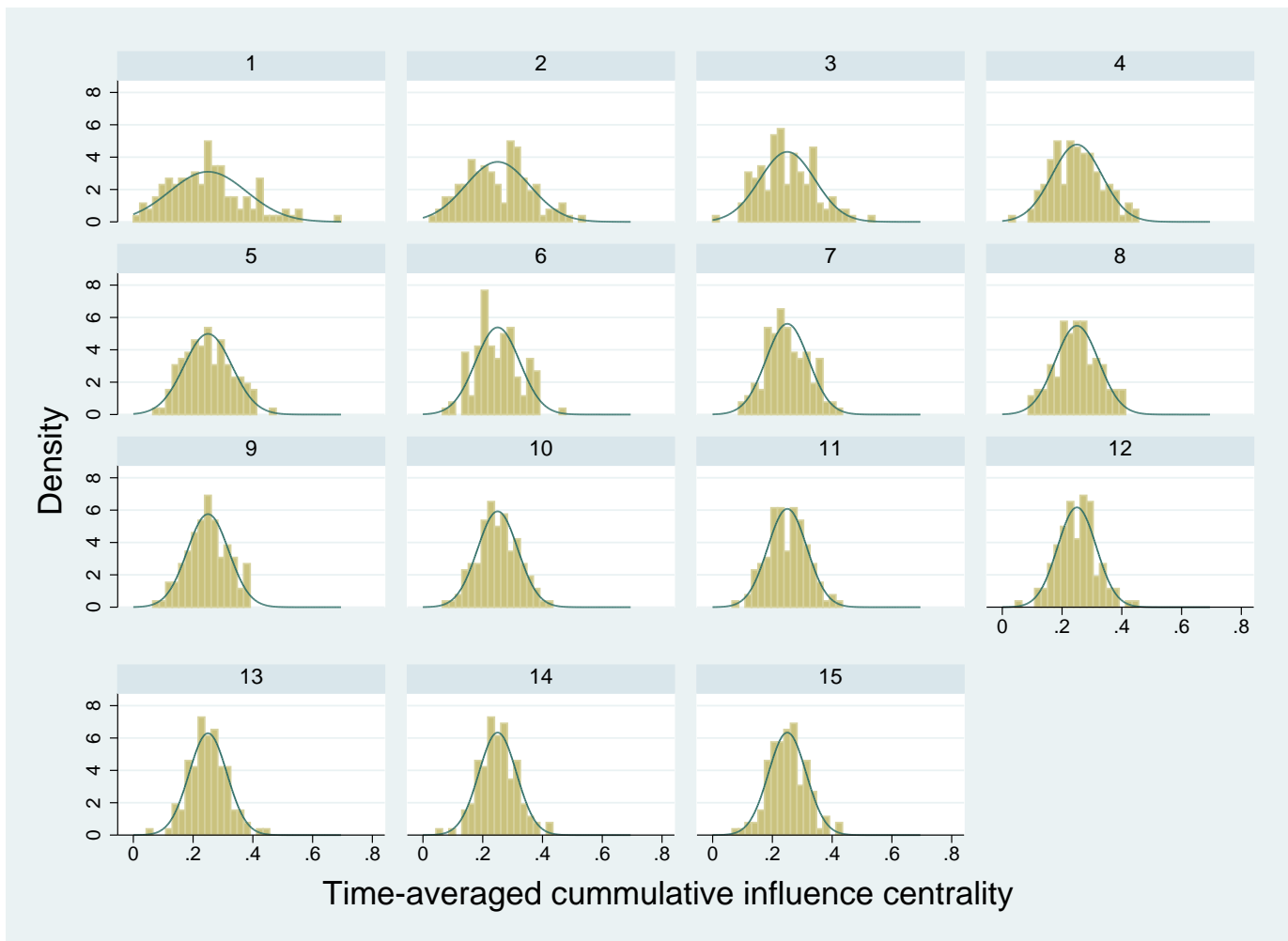

Figure 4: Evolution of the distributions of time-average influence centralities $T_{i}(s)=\frac{1}{s} \sum_{t=1}^{s} C_{i}(t)$ along the issue sequence

strong $(\rho=0.769)$. With such an association, the reflection mechanism should manifest itself as a fixed effect in which an individual's influence centrality on issue $s$ predicts the individual's influence centrality on issue $s+1$ along the issue sequence $s=1,2, \ldots, 15$. Table 3 indicates that such continuity exists in these data. In particular, note that the effect of $T_{i}(s)$ on $C_{i}(s+1)$ increases along the sequence $0.176+0.049 s, s=1,2, \ldots, 15$.

Henceforth, our attention is focused on the evolution of individuals' timeaverage influence centrality $T_{i}(s)=\frac{1}{s} \sum_{t=1}^{s} C_{i}(t)$ along the issue sequence. Figure 4 shows that the distribution of individuals' time-average influence centrality concentrates along the sequence. On each issue of the issue sequence, the means of these influence centrality distributions are invariant $(0.25)$.

Figure 5 displays the individual-level trajectories of each individual's cumulative centrality $\sum_{t=1}^{s} C_{i}(t)$ and time-average centrality $T_{i}(s)=\frac{1}{s} \sum_{t=1}^{s} C_{i}(t)$ along the issue sequence $s=1, \ldots, 15$ in each of the 30 groups. These trajectories show that the individuals in each group are accumulating influence centralities at different rates, and that their time-average centrality stabilizes to constant values along the issue sequence. Figure 6 shows that the probability of an individual's state of complete closure to influence on issue $s+1$ of the issue sequence increases with the individual's prior time-averaged influence centrality $T_{i}(s)$. Figure 7 shows that the frequency of instances of group members who are completely closed to influence is 

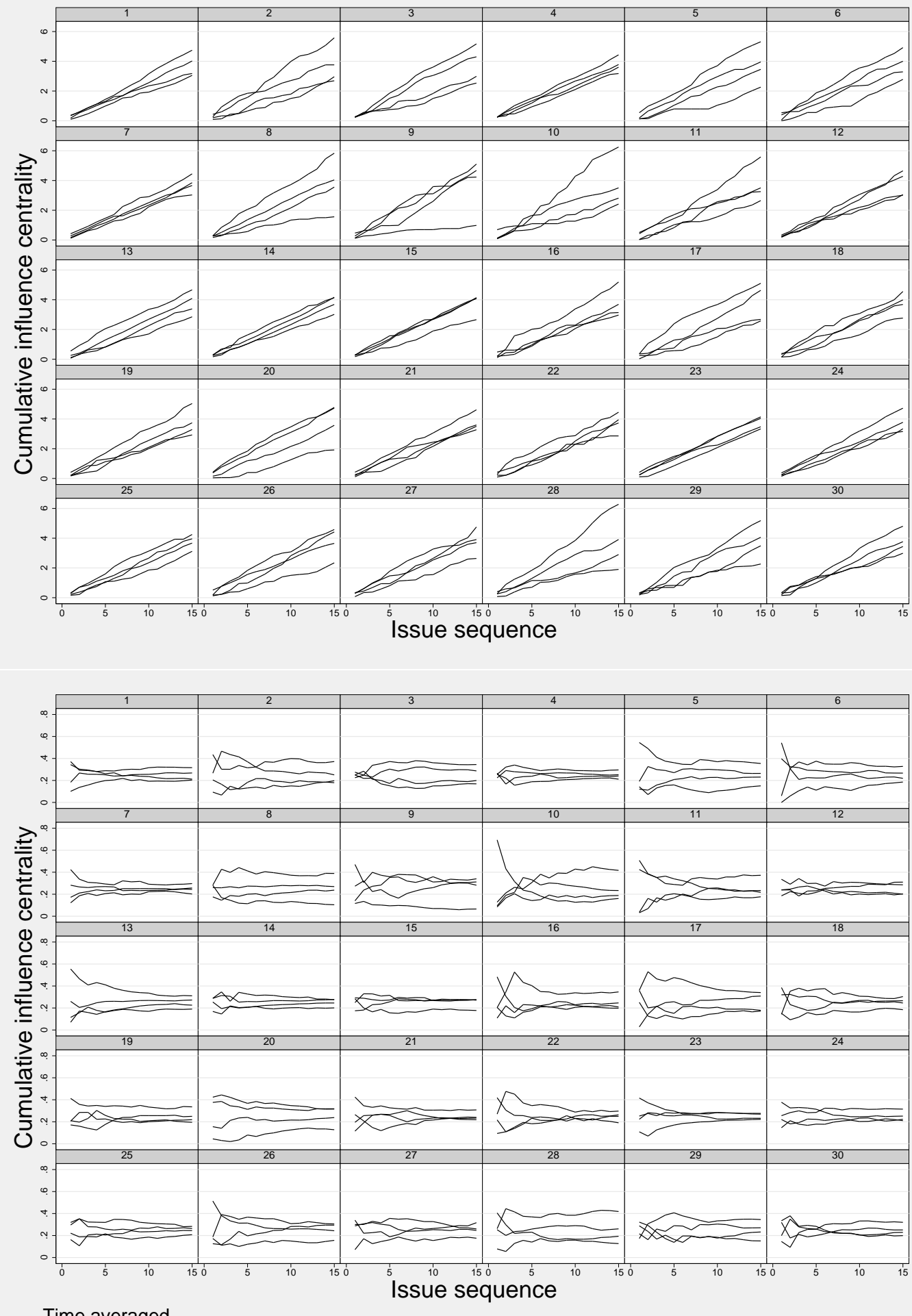

Time averaged

Figure 5: Evolution of individuals' cumulative influence centrality $\sum_{t=1}^{s} C_{i}(t)$ and time-average centrality $T_{i}(s)=\frac{1}{s} \sum_{t=1}^{s} C_{i}(t)$ for each individual in each of the 30 groups along the issue sequence. 


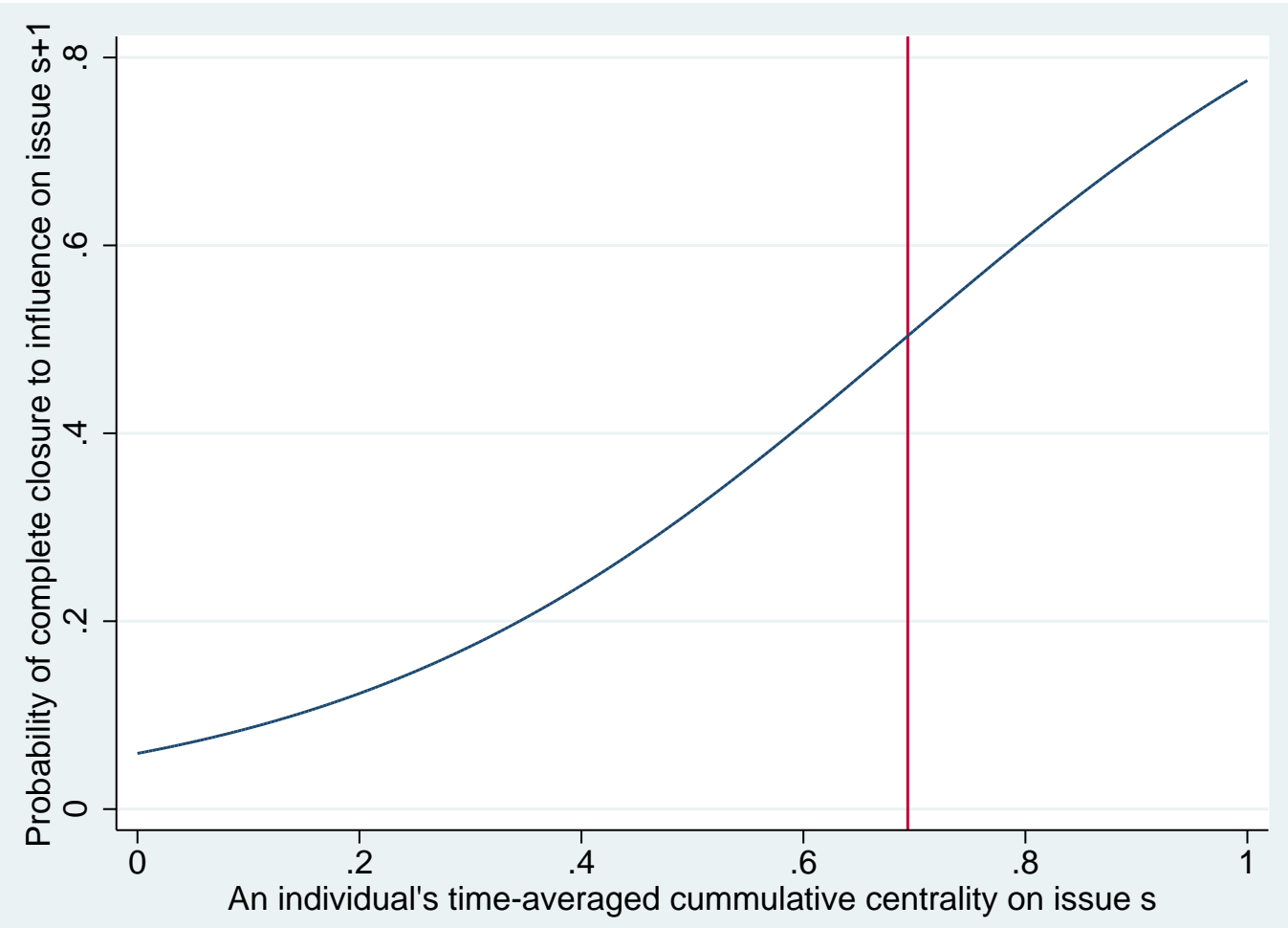

Figure 6: Prior time-averaged centrality $T_{i}(s)=\frac{1}{s} \sum_{t=1}^{s} C_{i}(t)$ of individual $i$ and the individual's probability of complete closure to influence $a_{i i}=1-w_{i i}=0$ on issue $s+1$. Balanced logistic random-intercept multilevel longitudinal design. Plot of estimated probabilities based on odds ratio estimates $\beta_{0}=0.063$ (s.e. $=0.018$ ), $p \leq 0.001 ; \beta_{1}=54.798$ (s.e. $=45.018$ ),$p \leq 0.001$. The vertical line indicates the maximum observed value of $T_{i}(s)$ in the dataset.

elevated along the issue sequence. In other words, the stabilizing relative differences of individuals' $T_{i}(s)$ centralities become increasingly indicative of the unequal rates at which individuals are accumulating centrality. Hence, the finding in Tables 2 and 3 on the increasing effect of $T_{i}(s)$ along the sequence.

\section{Group-level findings}

The observed adjustments of individuals' levels of closure to influence along an issue sequence, in response to their prior influence centralities, are consistent with the key prediction of the reflection mechanism. As illustrated in the introductory Figures 1-3, the structural implications of the reflection mechanism depend on the structural instability of the configuration of interpersonal influences (whoinfluences-whom) in the networks that are formed along the group's issue sequence. In this section, we present group-level findings on the observed evolution of influence systems along an issue sequence. The analysis is now focused on the group as a social unit and the prediction that its evolution, which may be repetitively disturbed, on average alters the group's network structure toward a generic structure-a unilateral network with one member who is closed to influence on a specific issue, and 


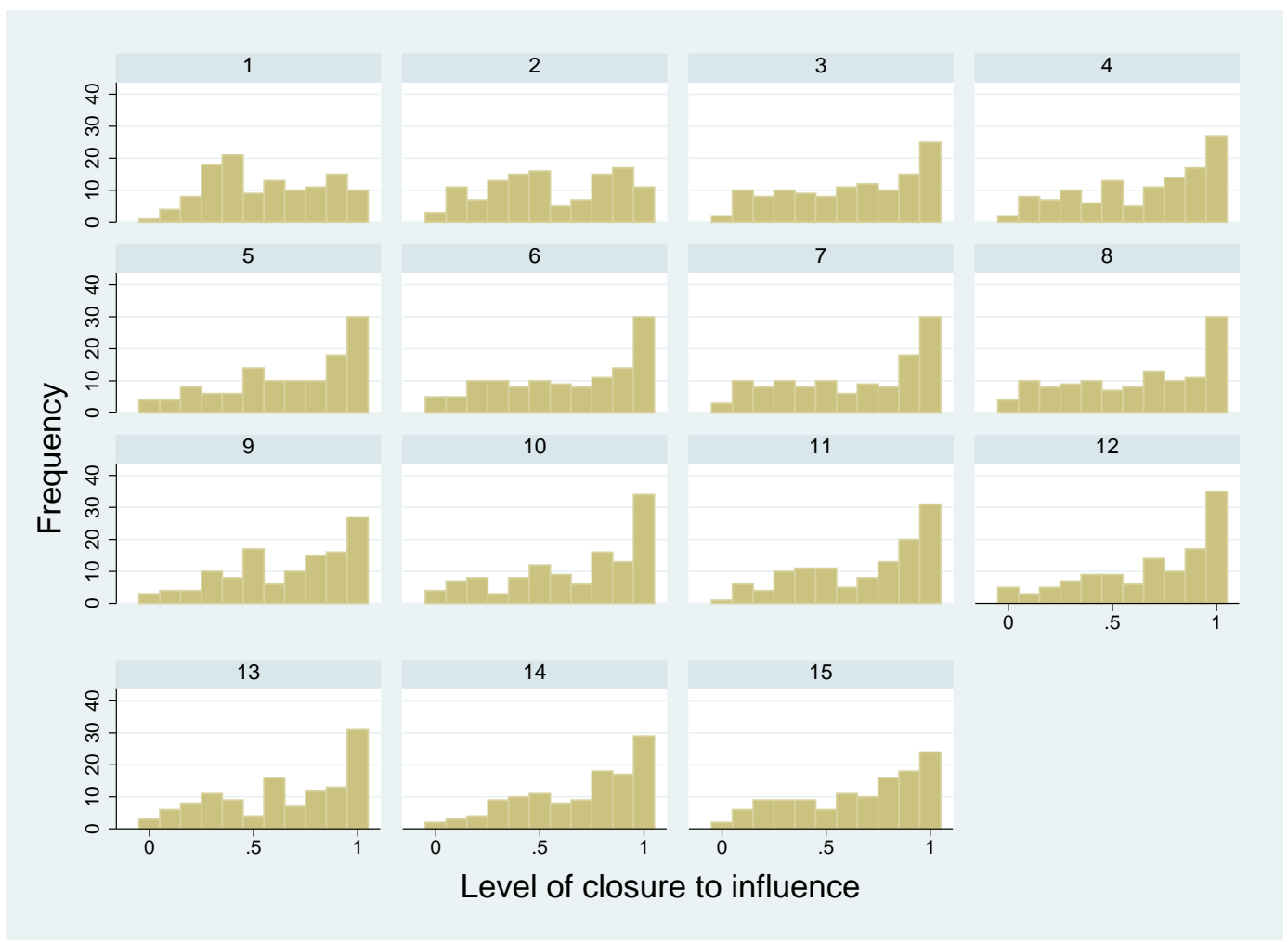

Figure 7: Histograms of the 120 individuals' levels of closure to influence $w_{i i}$ along the issue sequence, indicating the increase in frequency of individual states of complete closure to influence.

who influences directly or indirectly all others, who are open to influence on the issue. Structural disturbances may alter who that closed member is. The generic outcome is a consensus on the initial opinion of the group's closed member, as opposed to a compromise position or unreconciled disagreement. The expectation is a movement on average toward a system in which the group as a social unit adjusts its matrix of influence allocations, from issue to issue, to accommodate instances of a strongly held initial opinion and reach consensus.

\section{Task completion times}

Table 4 shows that the average observed time to task completion (minutes) declines along the issue sequence. Figure 8 displays the estimated expected values and observed mean times. Note the slight rise near the end of sequence. With recruited subjects who are dealing with an issue sequence that has a known length, in an experiment consuming hours of time, such near-end-of-sequence effects are not surprising, and we will see such effects in other findings presented below. However, we will also see that the point where the observed rise occurs corresponds to a threshold in the evolution of group structure at which a small difference of network density has a large effect on the topology of the group's influence network.

Table 4 also evaluates the association of groups' observed time to task completion and the $\ln (\mathrm{stab})$ predictor of the number of opinion updates required to reach a 
Table 4: Evolution of observed time (minutes) to task completion (reaching a state of consensus or irreconcilable disagreement) along the issue sequence $s=1,2, \ldots, 15$. Standard errors are in parentheses.

\begin{tabular}{lrr}
\hline & \multicolumn{1}{c}{ (a) } & (b) \\
\hline$s$ & $-0.926^{*}$ & $-0.901^{*}$ \\
$s^{2}$ & $(0.205)$ & $(0.191)$ \\
& $0.039^{\dagger}$ & $0.041^{*}$ \\
initial opinion range & $(0.012)$ & $(0.012)$ \\
& & $0.058^{*}$ \\
F-J ln(stab) predictor & $(0.009)$ \\
Constant & & $1.736^{*}$ \\
& & $(0.449)$ \\
R-square(overall) & $16.272^{*}$ & $8.670^{*}$ \\
\hline
\end{tabular}

Notes: $* p \leq 0.001,+p \leq 0.01$; balanced random-intercept fixed effects longitudinal design; STATA 12 xtreg implementation; $n=450$.

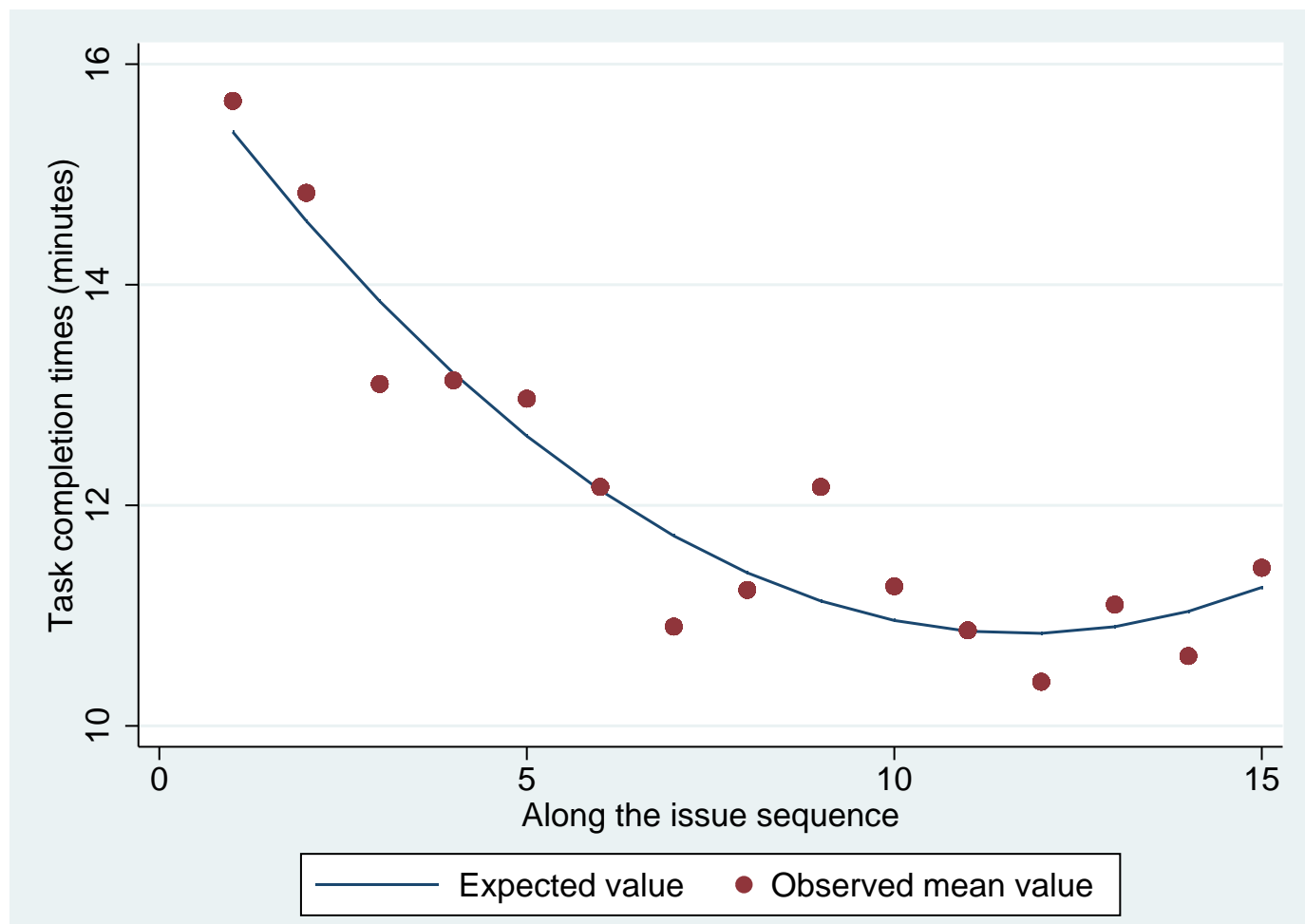

Figure 8: Trajectory of time to task completion along the issue sequence estimated from the model(a) estimates of Table 4. 


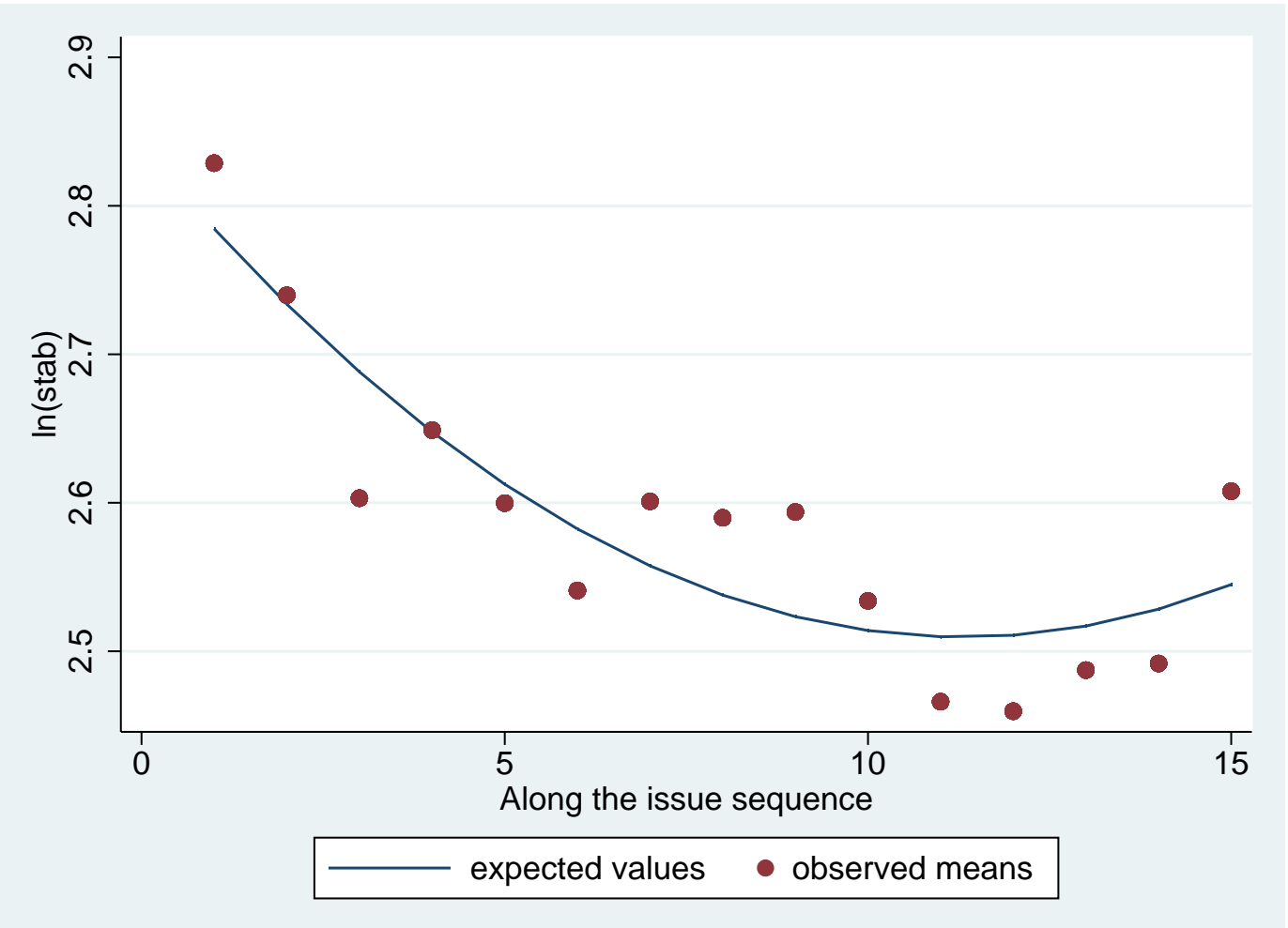

Figure 9: Evolution of $\ln (\mathrm{stab})$ along the issue sequence. Balanced random-intercept fixed effects longitudinal design; STATA 12 xtreg implementation; $n=450$; plot of expected values based on the estimated $\beta_{\text {constant }}=$ 2.840 (s.e. $=0.075), p \leq 0.001 ; \beta_{s}=-0.059$ (s.e. $\left.=0.022\right), p \leq 0.01, \beta_{s^{2}}=0.003$ (s.e. $=0.001$ ),$p \leq 0.05$; R-square (overall) 0.027 .

near settled state of opinions on a specific issue. The statistical significance of this time-to-stability predictor is additional support for the Friedkin-Johnsen dynamical system specification. The measure is strictly a function of the structure of the influence network associated with the group's matrix of allocated influence AW $(s)$ on a specific issue. As such, the rate of convergence that it indicates may serve as a portal into an investigation of the structural evolution of influence networks along the issue sequence. The next section of findings evaluates whether a structural evolution occurs in these data.

\section{Structural evolution metrics}

The $\ln (\mathrm{stab})$ measure of the rate of convergence to a state of settled opinions is strictly a function of a group's influence allocation matrix AW on a specific issue. If this metric declines along the issue sequence, then such a decline indicates systematic structural adjustments of AW that improve the efficiency of an evolving influence system. Figure 9 shows that the convergence time of the system, i.e., $\ln (\mathrm{stab})$, does decline along the issue sequence. Systematic structural changes are occurring in the midst of substantial structural disturbances. 
Table 5: $\ln (s t a b)$ predictors. Standard errors are in parentheses.

\begin{tabular}{|c|c|c|}
\hline & (a) & (b) \\
\hline Dominant eigenvalue of AW & $\begin{array}{c}2.932 * \\
(0.086)\end{array}$ & $\begin{array}{c}3.309^{*} \\
(0.081)\end{array}$ \\
\hline $\begin{array}{l}\text { Connectivity category of AW } \\
\text { weak }\end{array}$ & $\begin{array}{r}0.548^{+} \\
(0.054)\end{array}$ & \\
\hline unilateral & $\begin{array}{c}0.507^{*} \\
(0.063)\end{array}$ & \\
\hline strong & $\begin{array}{c}0.411^{*} \\
(0.077)\end{array}$ & \\
\hline (Baseline: disconnected) & & \\
\hline Influence density of AW & $\begin{array}{c}0.004^{*} \\
(0.001)\end{array}$ & \\
\hline Constant & $\begin{array}{c}0.915^{*} \\
(0.047)\end{array}$ & $\begin{array}{c}1.496^{*} \\
(1.374)\end{array}$ \\
\hline R-square(overall) & 0.885 & 0.815 \\
\hline
\end{tabular}

Elementary properties of AW predict $88.5 \%$ of the variance of $\ln (\mathrm{stab})$, and these elementary properties evolve along the issue sequence. Table 5 reports the findings on these $\ln (\mathrm{stab})$ predictors. The dominant eigenvalue is the major variable determining the rate of influence system convergence to stability. Figure 10 plots the dominant eigenvalue's trajectory.

In Table 5, the $\ln (\mathrm{stab})$ rate of convergence is also associated with the density of nonzero allocated interpersonal influences $i \stackrel{a_{i i} w_{i j}>0}{\longrightarrow} j, i \neq j$. The number of iterations to convergence increases with the network's density. Greater network density on average dampens the values of the $i \stackrel{a_{i i} w_{i j}}{\longrightarrow} j, i \neq j$ direct influences, and the values of the direct and indirect influence flows that are adjusting group members' opinions. Figure 11 shows that network density declines along the issue sequence. The issue sequence effect on network density $-4.043+2 \times 0.188 \mathrm{~s}$ indicates that the rate of its decline along the issue sequence diminishes, and it is zero at $s=10.766$. The context in which this decline is occurring is important. It a sociological axiom that the strength of an interpersonal relation increases with the frequency of interpersonal interaction. Its widely accepted correlate is that small face-to-face groups, with sustained interpersonal interactions, tend to be composed of strong interpersonal ties and high levels of network density. However, in task-oriented groups, the sustained engagement along an issue sequence appears to have the opposite effect on interpersonal influence allocations. 


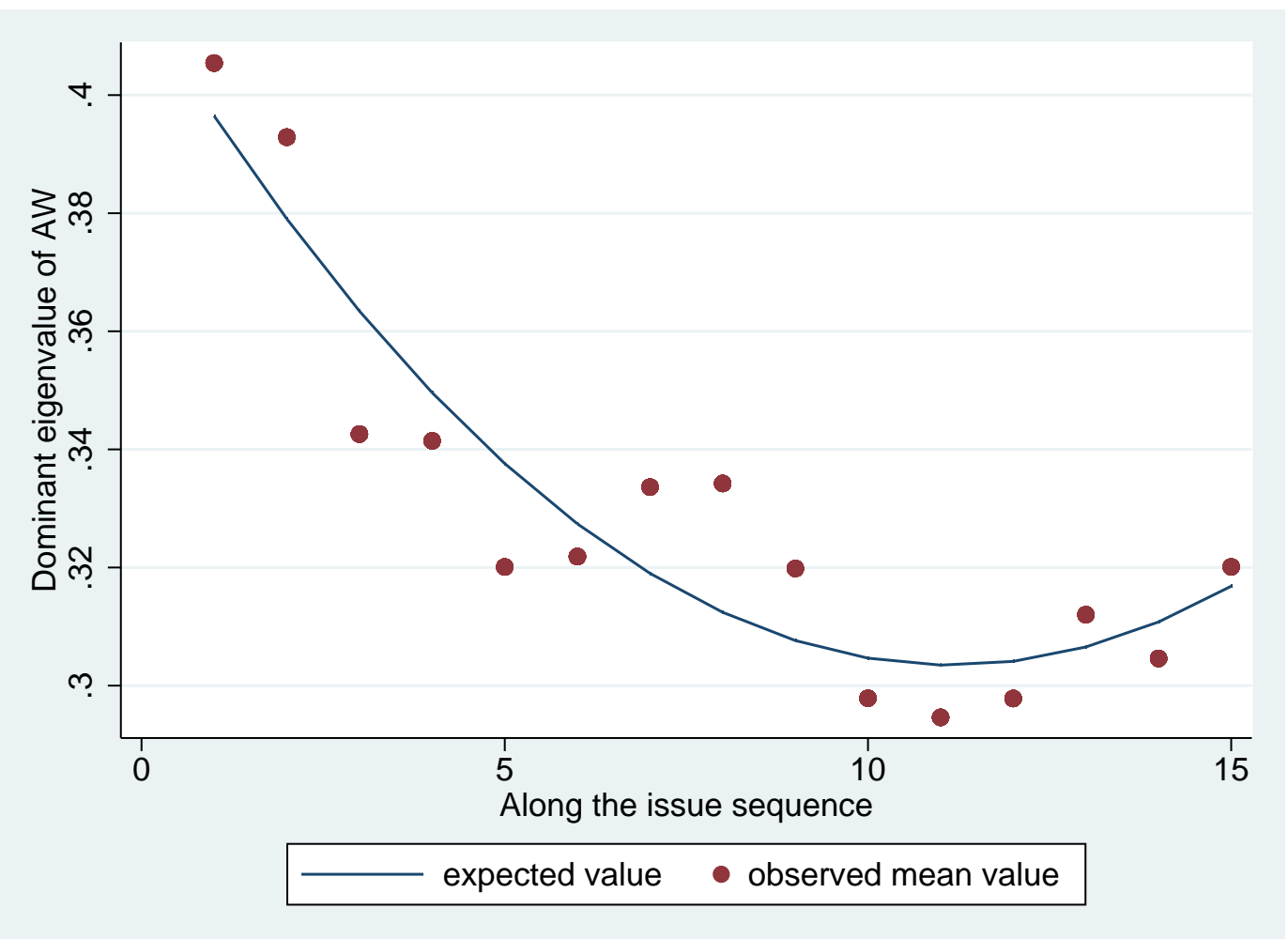

Figure 10: Evolution of the dominant eigenvalue of AW. Balanced random-intercept fixed effects longitudinal design; STATA 12 xtreg implementation; $n=450$ : plot of expected values based on the estimated $\beta_{\text {constant }}=$ 0.0 .416 (s.e. $=0.020$ ), $p \leq 0.001 ; \beta_{s}=-0.020$ (s.e. $=0.006$ ), $p \leq 0.001, \beta_{s^{2}}=0.001$ (s.e. $=0.0004$ ), $p \leq 0.01$; R-square (overall) 0.043 .

The estimated expected values of network density decline from $84.43 \%$ at $s=1$ to $66.53 \%$ at $s=11$ and then rise to $69.89 \%$ at $s=15$. In groups of size $n=4$, this decline alters the distribution of network topologies. The possible binary networks corresponding to the nonzero off-diagonal elements of AW include all possible $2^{16}=65,536$ networks. ${ }^{4}$ In the possible and observed networks, the network density must either $\leq 0.50,0.58 \overline{3}, 0.66 \overline{6}, 0.750,0.83 \overline{3}$, or $>0.8 \overline{3} .{ }^{5}$ Table 6 shows that the above estimated decline of network density to $66.53 \%$ at $s=11$ is a threshold value above which disconnected and weak topologies cannot exist in a $n=4$ group, and that loss of one additional positive edge dramatically elevates the proportion of unilateral networks. It is at this threshold where the observed rate of decline of network density is closest to zero, and it at this threshold where additions and subtractions of edges have a large affect on network topology.

The important implication is that the observed structural evolution of AW toward the $s=11$ domain of network density is a movement toward a state of diminished network density in which unilateral connectivity prevails. In this domain of density, only those unilateral structures with one completely closed-toinfluence member and three completely open-to-influence members are consistent with reaching an exact consensus under the condition of all different initial opinions. 


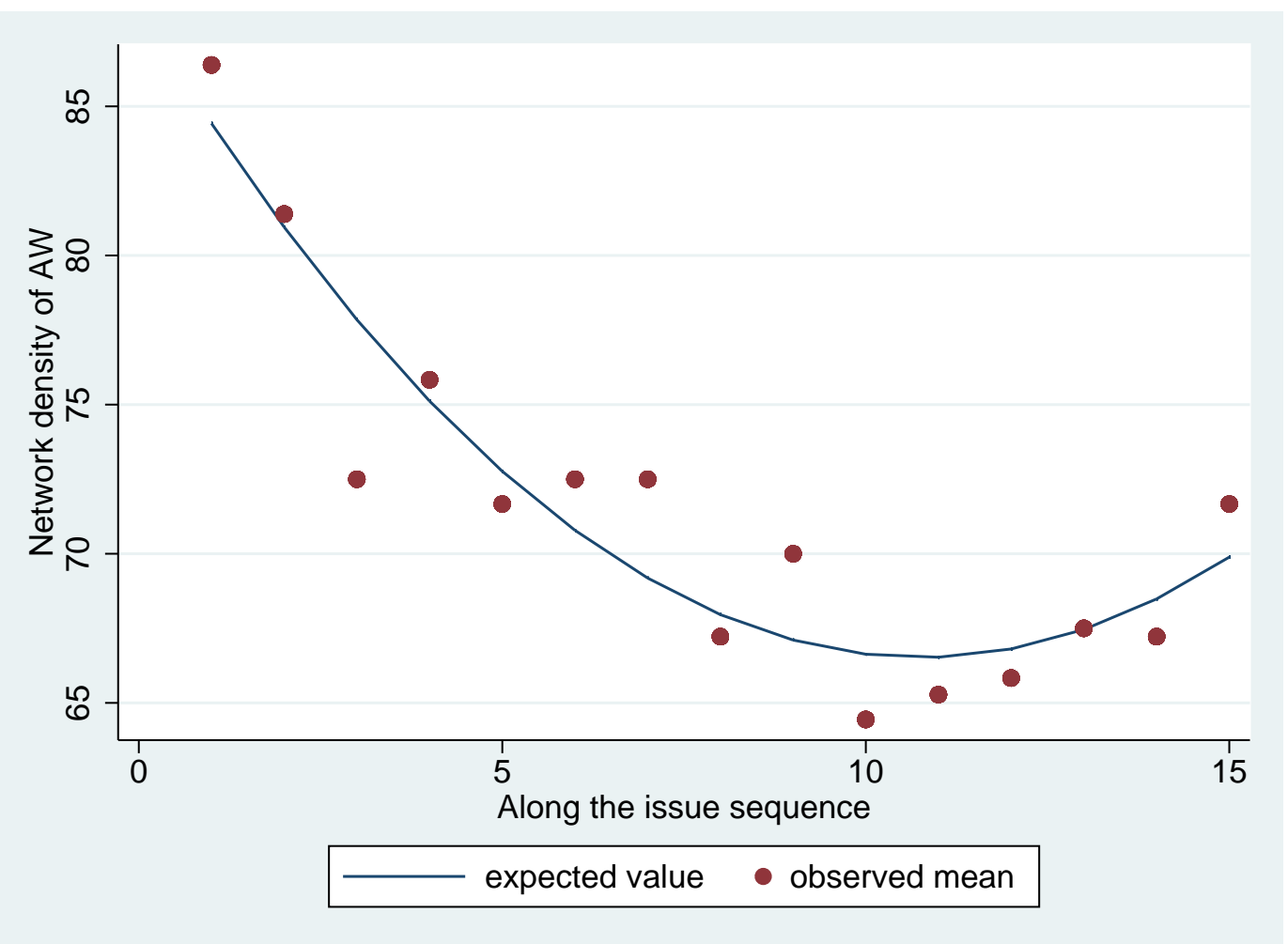

Figure 11: Evolution of the network density of AW. Density scaled $0-100 \%$. Balanced random-intercept fixed effects longitudinal design; STATA 12 xtreg implementation; $n=450$. Plot of expected values based on the estimated $\beta_{\text {constant }}=88.284$ (s.e. $=3.075$ ), $p \leq 0.001 ; \beta_{s}=-4.043$ (s.e. $=0.884$ ), $p \leq 0.001, \beta_{s^{2}}=0.188$ (s.e. $=$ $0.054), p \leq 0.001 ;$ R-square (overall) 0.053 .

In our data, the probability of reaching consensus does not alter along the issue sequence; it appears to be an imperative even under the manipulated condition of low pressure to achieve consensus. The 450 specific-issue resolutions are nearly equally distributed into three types: $36.89 \%$ unreconciled disagreement, $31.78 \%$

Table 6: Network density constraints on the distributions of network connectivity categories in $n=4$ groups in the population of all possible $2^{16}=65,536$ directed graphs.

\begin{tabular}{lccccc}
\hline Density & $\leq 0.50$ & $0.58 \overline{3}$ & $0.66 \overline{6}$ & 0.750 & $>0.75$ \\
\hline & $\%$ & $\%$ & $\%$ & $\%$ & $\%$ \\
Disconnected & 16.27 & 0.23 & 0 & 0 & 0 \\
Weak & 26.61 & 0.70 & 0 & 0 & 0 \\
Unilateral & 51.45 & 51.98 & 15.76 & 2.80 & 0 \\
Strong & 5.67 & 47.09 & 84.24 & 97.20 & 100 \\
\hline Total \% & 100 & 100 & 100 & 100 & 100 \\
Total $n$ & 25,376 & 27,456 & 7,920 & 4,576 & 208 \\
\hline
\end{tabular}




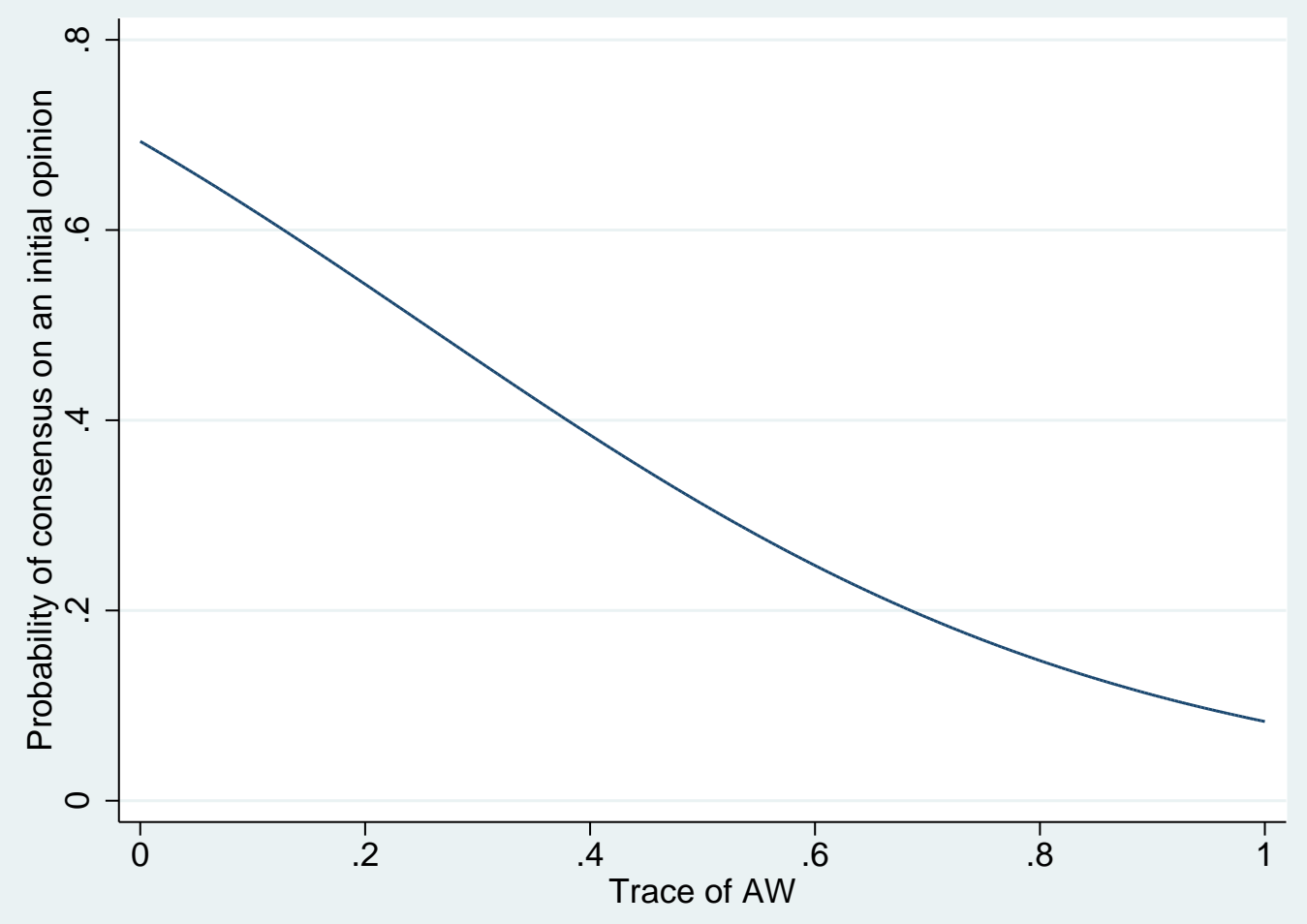

Figure 12: Estimated probability of group consensus on an initial opinion conditional on the trace of AW, trace $(\mathbf{A W})=\sum_{i=1}^{n} a_{i i} w_{i i}$. Balanced random-effects logistic regression longitudinal design; STATA 12 xtlogit implementation; $n=450$. Plot of estimated probabilities based on the odds ratio estimates $\beta_{\text {constant }}=2.260$ $($ s.e. $=0.899), p \leq 0.05, \beta_{\text {trace }}=0.0421($ s.e. $=0.026), p \leq 0.001, \log$ likelihood -256.150 .

consensus on an initial opinion, and $31.33 \%$ compromise consensus on an opinion that is not one of the group members' initial opinions. The size of the group's range of initial opinion does not alter along the issue sequence. What alters is the group's structural foundation of consensus production.

Figure 12 shows that the trace of $\mathbf{A W}$ is associated with the probability of a group consensus on an initial opinion. The estimated probabilities rise from 0.092 to 0.693 as the trace(AW) approaches 0 . Figure 13 shows that the trace declines to a threshold value near 0.50 along the issue sequence. In the F-J model of single issue dynamics, in a group with all different initial opinions on an issue (the majority of cases in the present data), reaching consensus on one member's initial opinion can only occur when one member's $w_{i i}=1$ and all other members' $w_{j j}=0$. In the small groups of this study, this circumstance is associated with the probability that a group will reach consensus on the initial opinion of the uniquely closed member of the group. 


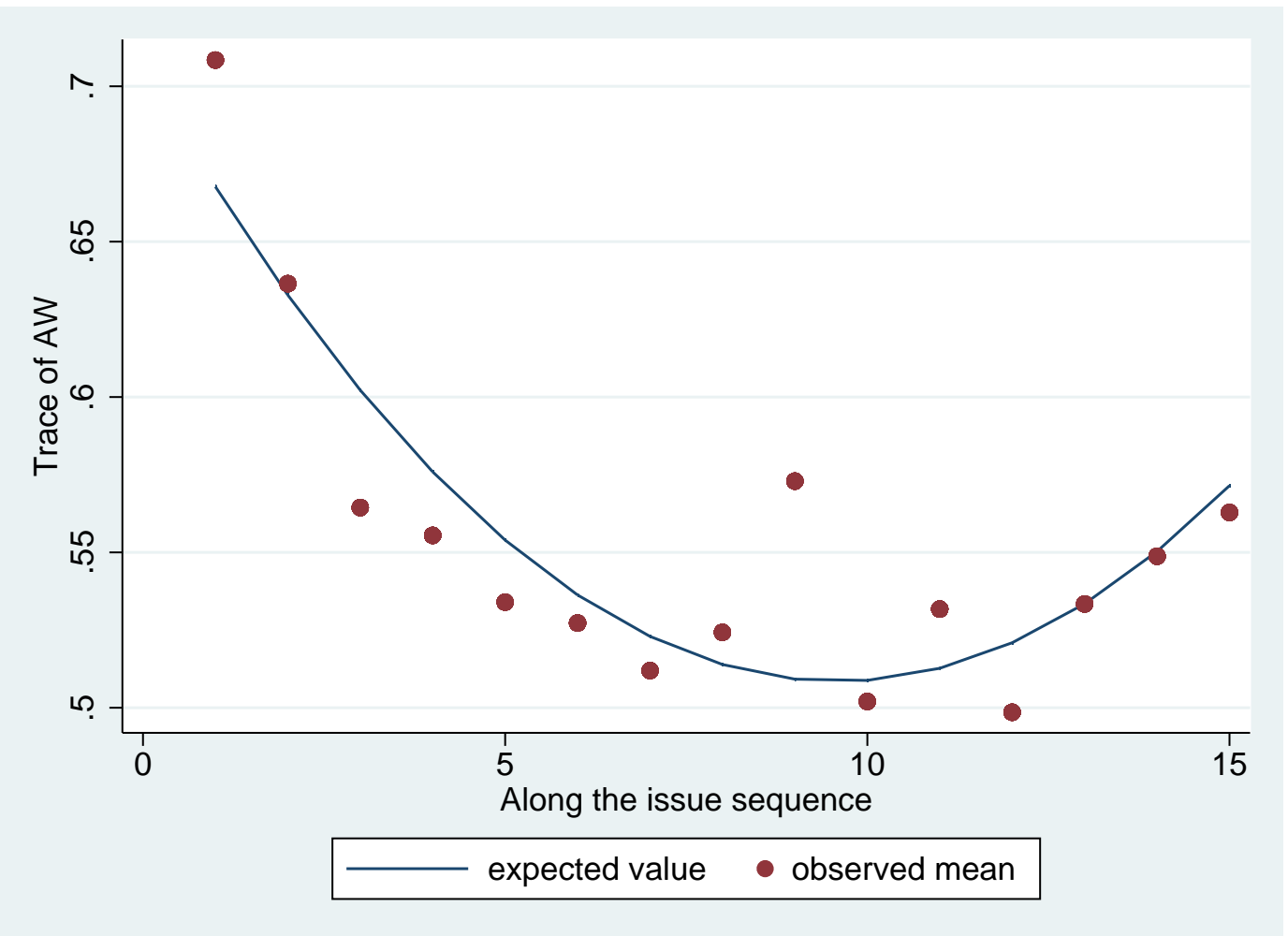

Figure 13: Evolution of the trace of $\mathbf{A W}$, trace $(\mathbf{A W})=\sum_{i=1}^{n} a_{i i} w_{i i}$. Balanced random-intercept fixed effects longitudinal design; STATA 12 xtreg implementation; $n=450$. Plot of expected values based on the estimates $\beta_{\text {constant }}=0.707$ (s.e. $=0.031$ ), $p \leq 0.001 ; \beta_{s}=-0.041$ (s.e. $=0.009$ ), $p \leq 0.001, \beta_{s^{2}}=0.0 .002$ (s.e. $=0.0 .001$ ), $p \leq 0.001 ;$ R-square (overall) 0.043 .

\section{Discussion}

Networks constituted by the elementary events of $i \rightarrow j$ allocations of influence may present highly idiosyncratic social structures that do not neatly conform to idealized forms of social organization. The influence systems that a group assembles on specific issues, along an issue sequence, may vary from issue to issue. Both failure and success in reaching consensus in a group discussion regularly occur, at least in our data where in the ensemble of 450 group discussions investigated $36.89 \%$ of the discussions failed to reach consensus. It is, therefore, surprising that any structural trajectories of group evolution along issue sequences have been detected.

Our work on issue sequences is an effort to advance the field of work on opinion dynamics. This is a field with broad fundamental significance to which investigators from a variety of disciplines are contributing. Although we have a rapidly accumulating literature on single-issue opinion dynamics, we know little about group dynamics over issue sequences. Our findings indicate that a fruitful line of formalization may be developed on the evolution of interpersonal influence systems along issue sequences. The reflection mechanism upon which we have focused is 
likely to be one mechanism, in a suite of evolutionary mechanisms, that operate to alter influence systems.

The idea that individuals' self-appraisals are influenced by others' appraisals of them (Cooley, 1902) is a classic postulate of a ubiquitous social construction of individuals' self-esteem, self-efficacy, and locus-of-control. The postulate assumes that individuals are generally susceptible to interpersonal influence and draws attention to social groups in which individuals are embedded. We believe that this mechanism is activated in deliberative groups in which individuals are appraising the displayed opinions of group members on specific issues in an effort to reach a consensus. In this collaborative task, positive and negative appraisals of displayed opinions are significant events, as are the influence centralities of group members, where these centralities correspond to the net relative influences of each group member's initial opinion on the settled opinions of the group's members. The behavior of individuals in deliberative groups (assertive advocacy vs. silence, confidence vs. uncertainty, intransigence vs. accommodation) may be altered as adaptive responses to prior realized influence in their groups. The key scope condition of the postulated reflection mechanism is a consensus seeking group in which failures to reach consensus are viewed as social problems. In such groups, accommodation is a pro-social contribution to group solidarity, unsupported intransigence on an issue is anti-social behavior, and supported intransigence is a bestowed honor.

The reflection mechanism sets up a potential issue-sequence evolution of individuals' influence centralities. Inequalities of prior influence centrality alter group member's expectations of future influence in the group and adjust their levels of closure-openness to interpersonal influence on the next issue considered by the group. If a strongly connected influence network is formed on the next issue, then such adjustments alter all flows of influence in the group and, more generally, all flows of influence that involve the individuals whose levels of closure have been altered. Hence, what may appear to be a radically micro-level mechanism, is just the opposite. Individuals' displayed levels of closure-openness to influence are group-specific manifestations of their self-appraised expected influence in a group, and such appraisals may have system-level implications. The implications are illustrated in the computational analyses of the mechanism. Inequalities of influence centrality are reinforced, and unequal linear trends of individuals' centralities present a brutal mathematical logic. A unique maximum trend line for one individual over a sufficiently lengthy sequence of issues must eventually force a concentration of relative total influence on that group member. Our model and empirical findings suggest that, in the opinion dynamics of collaborative groups, the reflection mechanism is a natural micro-foundation of unequal linear trends of individuals' influence centralities.

Our empirical findings on group-level structural metrics indicate that group social structure evolves along an issue sequence in the midst of disturbances. The particular metrics of this evolution that we have investigated are constrained by our model of the influence system that alters group members' opinions. As a theory driven investigation, our group-level analysis is oriented to an evaluation of whether the group-level implications of the reflection mechanism appear in the 30 groups investigated. The possible theoretical manifestations and implications 
of the mechanism include both simple and complex (e.g., oscillatory) trajectories of group-level evolution. Our group-level findings are signals, in the midst of structural disturbances, of an evolution of groups along an issue sequence, on multiple dimensions of group structure, that is consistent with the existence of the reflection mechanism. Amidst substantial structural disturbances, the detected structural trajectories are toward the predicted attractor system. It is a system that expeditiously reaches consensus based on influence allocation adjustments that are accommodations to the displayed strongly held opinion of a member with a prior record of influence centrality. In the absence of substantial structural disturbances, the trajectory is toward a system with one dominant individual (a directive leader) whose initial opinions are accommodated. These findings have a straightforward application to the literature on groupthink, and we have located a discussion of this application in Section S4 of the online supplement for those readers who may have an interest in it.

\section{Notes}

1 A rough indicator of the emergence of the field is indicated by a Google Scholar search based on the key words "opinion dynamics". The number articles appearing in the periods 1900-1990, 1991-2000, 2001-2010, and 2011-2015 are 42, 136, 1,900, and 3,100 respectively.

2 An additional important technical classification is whether the network is periodic or aperiodic. It is aperiodic if in $\mathcal{G}$ there is no integer greater than one that divides the length of every cycle of the graph. Whether it is aperiodic or not is sometimes difficult to determine. A sufficient condition is the following: a strongly connected AW is aperiodic if at least one $0<a_{i i} w_{i i}<1$ exists, that is, a loop $i \rightarrow i$ edge in $\mathcal{G}$. Aperiodicity is important because without it, the $\lim _{k \rightarrow \infty}(\mathbf{A W})^{k}$ does not exist, and the matrix of total influence $\mathbf{V}$ for the influence system does not exist.

3 Where individual-level findings are reported, the statistical model employed is maximumlikelihood multilevel random-intercept linear regression with robust standard errors, or multilevel random-intercept logistic regression. The implementation is STATA 12's xtmixed and xtmelogit procedures. Where group-level findings are reported, the statistical model employed is STATA 12's xtreg procedure.

4 This matrix allows all-zero rows.

5 The possible number of nonzero off-diagonal elements $\mathbf{A W}$ is 12 in a $n=4$ group. For the $6-12$ number of possible nonzero elements of the matrix, the network densities are $0.50,0.58 \overline{3}, 0.66 \overline{6}, 0.750,0.83 \overline{3}, 0.916 \overline{6}$, and 1 , respectively.

\section{References}

Bonacich, P. 1987. "Power and centrality: a family of measures." American Journal of Sociology 92:1170-1182.

Cooley, C. H. 1902. Human Nature and the Social Order. New York, NY: Charles Scribner Sons. 
DeGroot, M. H. 1974. "Reaching a consensus." Journal of the American Statistical Association 69:118-121.

Freeman, L. C. 1979. "Centrality in networks: I. Conceptual clarification." Social Networks 1:215-239.

French, J. R. P. 1956. "A formal theory of social power." Psychological Review 63:181-194.

Friedkin, N. E. 1991. "Theoretical foundations for centrality measures." American Journal of Sociology 96:1478-1504.

Friedkin, N. E. 2011. "A formal theory of reflected appraisals in the evolution of power.” Administrative Science Quarterly 56:501-529.

Friedkin, N. E. and E. C. Johnsen. 2011. Social Influence Network Theory: A Sociological Examination of Small Group Dynamics. New York, NY: Cambridge University Press.

Harary, F. 1959. "A criterion for unanimity in French's theory of social power." In Studies in Social Power, edited by D. Cartwright, pp. 168-182. Ann Arbor, MI: Institute for Social Research.

Hubbell, C. H. 1965. "An input-output approach to clique identification." Sociometry 28:377-399.

Jia, P., A. Mirtabatabaei, N. E. Friedkin, and F. Bullo. 2015. "Opinion dynamics and the evolution of social power in influence networks." SIAM Review 57:367-397.

Katz, L. 1953. "A new index derived from sociometric data analysis." Psychometrika 18:39-43.

Acknowledgements: We thank the Editor and Associate Editors of this journal for their cogent comments. This material is based upon work supported by, or in part by, the U. S. Army Research Laboratory and the U. S. Army Research Office under grant numbers W911NF-15-1-0577, W911NF-15-1-0274, and W911NF-09-0001. The content of the information does not necessarily reflect the position or the policy of the Government, and no official endorsement should be inferred.

Noah E. Friedkin: Center for Control, Dynamical Systems and Computation, University of California, Santa Barbara. E-mail: friedkin@soc.ucsb.edu.

Peng Jia: Center for Control, Dynamical Systems and Computation, University of California, Santa Barbara. E-mail: pjia@engineering.ucsb.edu.

Francesco Bullo: Center for Control, Dynamical Systems and Computation, University of California, Santa Barbara. E-mail: bullo@engineering.ucsb.edu. 